\title{
Multiple scales in Pati-Salam unification models
}

\author{
Florian Hartmann, Wolfgang Kilian and Karsten Schnitter \\ Department Physik, Universität Siegen, \\ 57068 Siegen, Germany \\ E-mail: hartmann@physik.uni-siegen.de, kilian@physik.uni-siegen.de, \\ schnitter@tp1.physik.uni-siegen.de
}

ABSTRACT: We investigate models where gauge unification (GUT) proceeds in steps that include Pati-Salam symmetry. Beyond the Standard Model, we allow for a well-defined set of small representations of the GUT gauge group. We show that all possible chains of PatiSalam symmetry breaking can be realized in accordance with gauge-coupling unification. We identify, in particular, models with unification near the Planck scale, with intermediate left-right or SU(4) quark-lepton symmetries that are relevant for flavor physics, with new colored particles at accessible energies, and with an enlarged electroweak Higgs sector. We look both at supersymmetric and non-supersymmetric scenarios.

KEYwords: Spontaneous Symmetry Breaking, GUT, Supersymmetric Effective Theories, Renormalization Group

ARXIV EPRINT: 1401.7891 


\section{Contents}

1 Introduction $\quad 2$

2 Pati-Salam symmetry 3

3 General setup 4

4 Higgs mechanism and supersymmetry 9

4.1 Generic superpotential 9

$\begin{array}{lll}4.2 & \text { Higgs mechanism and supersymmetry } & 10\end{array}$

5 Spectra and phenomenology 12

$\begin{array}{ll}5.1 \text { Mass matrix } & 12\end{array}$

5.2 Goldstone bosons 14

5.3 MSSM Higgs 15

5.4 The $F$ multiplet $\quad 16$

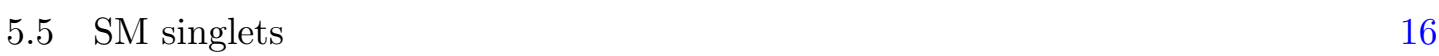

$\begin{array}{lll}5.6 & \text { Matter couplings } & 16\end{array}$

$\begin{array}{lll}5.7 & \text { Neutrino mass } & 17\end{array}$

6 Unification conditions $\quad 17$

7 Supersymmetric Pati-Salam models $\quad 19$

7.1 Extra mass parameters 20

$\begin{array}{ll}7.2 & \text { General overview }\end{array}$

$\begin{array}{lll}7.3 & \text { Class E } & 22\end{array}$

$\begin{array}{lll}7.4 & \text { Class F } 25\end{array}$

$\begin{array}{lll}7.5 & \text { Classes A to D } & 27\end{array}$

8 Pati-Salam models without supersymmetry 28

8.1 Class E 30

$\begin{array}{lll}8.2 & \text { Class F } & 30\end{array}$

8.3 Class A to D 31

9 Summary of models 33

10 Conclusions $\quad 34$

A Beta-function coefficients

B Vacuum expectation values and mass scales 36

$\begin{array}{ll}\text { C Model naming scheme } & 37\end{array}$

D Specific models $\quad 38$ 


\section{Introduction}

The Standard Model (SM) of particle physics organizes all known particles, including the Higgs boson, in a linear representation of the space-time and gauge symmetries. This representation is reducible and decomposes into several irreducible multiplets. Therefore, the SM Lagrangian contains a considerable number of free parameters. A unified theory (GUT) where the SM gauge symmetry is embedded in a larger gauge group can reduce the number of free parameters. Furthermore, there is a general expectation that a unified model of matter and interaction could explain some of the puzzles of particle physics, such as quark and neutrino flavor mixing, the smallness of neutrino masses, or dark matter.

Particularly promising GUT models are left-right symmetric extensions [1, 2], the PatiSalam model $[3,4]$, the SU(5) theory by Georgi and Glashow [5], or the combination of both in an $\mathrm{SO}(10)$ [6] or $E_{6}$ [7] gauge theory. In these GUTs, matter fields unify within each generation, separately. The SM Higgs sector is enlarged. Further fields, symmetries, or geometrical structure are required to explain the breaking of GUT symmetries and the emergence of the SM (or its minimal supersymmetric extension, MSSM) at energies directly accessible to us.

In this paper, we investigate various classes of Pati-Salam (PS) models. PS symmetry, with a discrete left-right symmetry included, is the minimal symmetry group which collects all matter fields of a single generation in one irreducible multiplet. As an extension of the SM, it is particularly interesting because it naturally accommodates multiple energy thresholds or scales that fill the large gap between the SM (TeV) scale and the scale of ultimate unification, presumably the Planck scale. Such models have been widely discussed in case of both supersymmetric [8-23] and non-supersymmetric models [2426]. PS models do not just exhibit multiple scales associated with symmetry breaking, but they can generate additional mass scales due to see-saw type patterns in the spectrum $[27,28]$.

We want to study the relation of symmetry breaking and intermediate mass scales in PS models in a suitably generic way while keeping the sector of extra (Higgs) fields below the GUT scale as compact as possible. To this end, we impose conditions on the spectrum of new fields, so we can describe the chain of symmetry breaking via a staged Higgs mechanism with only small representations of the GUT group. In this setup, we scan over the complete set of models and investigate the allowed ranges and relations of the various mass scales they can provide.

There are several specific questions that one can address by such a survey of models. First of all, some GUT models are challenged by the non-observation of proton decay, so we may ask whether complete unification, and thus the appearance of baryon-number violating interactions, can be delayed up to the Planck scale. Secondly, whether the origin of neutrino masses and flavor mixing could be associated with a distinct scale, decoupled from complete unification. Thirdly, whether there can be traces of unification, such as exotic particles, a low-lying left-right symmetry, or an enlarged Higgs sector, that may be observable in collider experiments or indirectly affect precision flavor data. 
By restricting our framework to economical spectra with only small representations (see section 3), we focus the study on phenomenologically viable models that look particularly attractive. Nevertheless, they span a large range of intermediate-scale configurations between the $\mathrm{TeV}$ and Planck scales. We do not impose extra technical conditions on the gauge-coupling running and matching $([9,12,25])$, so we can cover also corner cases where scale relations are tightly constrained.

After a short review of PS models in section 2, we lay out the detailed framework of our study in section 3. In section 4 we take a brief look at the staged Higgs mechanism, before we discuss the overall spectrum patterns in section 5 . In section 6 we implement the conditions of gauge coupling unification. Finally, section 7 and section 8 present generic properties and interesting details for the complete survey of PS models, divided in the two classes of supersymmetric and non-supersymmetric models respectively, before we draw conclusions.

\section{Pati-Salam symmetry}

By the label of Pati-Salam gauge group, we refer to the symmetry group

$$
\mathrm{SU}(4)_{C} \times \mathrm{SU}(2)_{L} \times \mathrm{SU}(2)_{R} \times Z_{\mathrm{LR}}
$$

The $\mathrm{SU}(4)_{C}$ factor is an extension of QCD with lepton number as fourth color, and there is a right-handed $\mathrm{SU}(2)_{R}$ symmetry analogous to the weak interactions. We impose an exact discrete symmetry $Z_{\mathrm{LR}}$ which exchanges $\mathrm{SU}(2)_{L}$ with $\mathrm{SU}(2)_{R}$, and left-handed with right-handed matter fields, respectively.

If we impose this symmetry on any generation of quarks and leptons, they combine to a single irreducible representation $\Psi_{L / R}$. Regarding the continuous gauge group only, this is a direct sum $\Psi_{L} \oplus \Psi_{R}$, but it is rendered irreducible by the left-right symmetry. ${ }^{1}$ In particular, this representation necessarily includes a right-handed neutrino field. The explicit embedding of the matter fields in the PS multiplet is as follows:

$$
\Psi_{L}=(\mathbf{4}, \mathbf{2}, \mathbf{1})=\left(\begin{array}{llll}
u_{r} & u_{g} & u_{b} & \nu \\
d_{r} & d_{g} & d_{b} & e
\end{array}\right)_{L}, \quad \Psi_{R}=(\overline{\mathbf{4}}, \mathbf{1}, \mathbf{2})=\left(\begin{array}{llll}
u_{r}^{c} & u_{g}^{c} & u_{b}^{c} & \nu^{c} \\
d_{r}^{c} & d_{g}^{c} & d_{b}^{c} & e^{c}
\end{array}\right)_{R} .
$$

Here the subscript $(r, g, b)$ are explicit color indices. This notation is compatible with the usual conventions for supersymmetric GUT models, where we will denote by $\Psi_{L / R}$ the corresponding superfield.

The set of gauge bosons of a PS gauge theory contains the eight QCD gluons and the weak $W_{L}$ triplet. On top of that, there are a $W_{R}$ triplet and seven extra gauge bosons of $\mathrm{SU}(4)_{C}$. The latter consist of six (vector) leptoquarks and one SM singlet that gauges the difference of baryon and lepton number, $B-L$. The SM hypercharge gauge boson emerges as a linear combination of the two neutral PS gauge bosons. The discrete $Z_{\mathrm{LR}}$

\footnotetext{
${ }^{1}$ Throughout this paper, we indicate such $Z_{2}$-irreducible direct sums by the subscript $L / R$, or simply write $\Psi$ for brevity.
} 
symmetry guarantees that the interactions of left-handed and right-handed fields coincide for all representations.

The PS group is a subgroup of $\mathrm{SO}(10)$, but does not contain $\mathrm{SU}(5)$. It is well known that the gauge couplings unify for a pure SU(5) GUT model, if supersymmetry is imposed. However, the recent discoveries about neutrino masses and mixing (see review article in [29]) suggest additional new physics at high energies. Concrete estimates tend to place the neutrino mass-generation scale below the GUT scale (either as direct mass scale [30] or via a see-saw mechanism [31]). We should expect multiple thresholds where the pattern of multiplets and symmetries changes. This idea fits well in the context of a PS symmetry and its breaking down to the SM.

Therefore, we investigate scenarios where a PS gauge symmetry is valid above some high energy scale. Below the PS scale, we allow for further thresholds where only a subgroup of PS is realized. One such subgroup is the minimal left-right symmetry $\mathrm{SU}(3)_{C} \times \mathrm{SU}(2)_{L} \times$ $\mathrm{SU}(2)_{R} \times \mathrm{U}(1)_{B-L} \times Z_{\mathrm{LR}}$. By construction, left-right symmetry breaking is associated with flavor physics, both in the quark and lepton sectors. ${ }^{2}$ Above the PS scale, we maintain the possibility of further unification to a GUT model. Examples are $\mathrm{SO}(10)$ [6] or a larger group such as $E_{6}[7]$.

PS models provide a partial explanation for the observed stability of the proton. $\mathrm{SU}(4)_{C}$ contains the $\mathrm{U}(1)_{B-L}$ subgroup, so $B-L$ is conserved. In analogy with QCD, lepton number as the fourth color - and thus baryon number - is conserved by all interactions that do not involve the totally antisymmetric tensor $\epsilon_{a b c d}$ with four color indices. Counting field dimensions, this excludes baryon-number violating interactions of fermions in the fundamental or adjoint representations of $\mathrm{SU}(4)_{C}$, at the renormalizable level. In a minimal PS extension of the SM, proton-decay operators are thus excluded or, at least, naturally suppressed. In particular, the terms of the MSSM which mediate proton decay via squark fields are inconsistent with PS symmetry. ${ }^{3}$

A field condensate that spontaneously breaks $\mathrm{SU}(4)_{C}$ to $\mathrm{SU}(3)_{C}$ carries lepton number, but no baryon number. Hence, PS breaking does not induce proton decay either, at the level of renormalizable SM or MSSM interactions. On the other hand, breaking PS symmetry down to the SM gauge groups can generate and relate the operators that provide neutrino Majorana masses and mixing in the lepton sector, as well as Yukawa terms and mixing in the quark sector.

\section{General setup}

In this paper, we consider both supersymmetric and non-supersymmetric versions of a PS gauge theory. We first investigate supersymmetric models, where the model space is more constrained. For this purpose, we assume $N=1$ supersymmetry to hold over the full

\footnotetext{
${ }^{2}$ In a left-right symmetric model, up- and down-type masses are degenerate, and all mixing angles can be rotated away.

${ }^{3}$ We note that supersymmetric PS models need not include $R$ parity for eliminating proton-decay operators. However, for more general extensions of the MSSM we have to discuss this issue (see section 5.4). For the sake of simplicity we imply $R$ parity for all our models.
} 
energy range above the $\mathrm{TeV}$ scale. Later we will also discuss the running of the gauge couplings in non-supersymmetric versions of the models.

Following a top-down approach, we put the highest scale under consideration at the Planck scale (we use $M_{\text {Planck }}=10^{18.2} \mathrm{GeV}$ ). In that energy range, gravitation is strong and a perturbative quantum field theory in four space-time dimensions is unlikely to be an appropriate description. Below this scale, gravitation becomes weak, and an effective weakly coupled four-dimensional gauge theory can emerge. This gauge symmetry may be the PS group. We suspect that at the highest energies there is a larger local symmetry (GUT) that contains PS as a subgroup. By $M_{\mathrm{GUT}}$ we denote the energy scale where this larger symmetry is broken down to PS. We note that $M_{\mathrm{GUT}}$ may coincide with $M_{\text {Planck }}$, and that the GUT theory need not be a perturbative field theory.

It is important to study possible mechanisms that can break a Planck-scale GUT symmetry down to the PS group. Unfortunately, few reliable and generic results are available. For instance, the survival principle that restricts the pattern of multiplets in the lowenergy effective theory [32-35] does not hold in supersymmetric theories [27, 28, 36]. In the vicinity of the Planck scale, all conceivable effects from string states, gravitation, extra dimensions, strong interactions, and possibly completely unknown principles need to be taken into account in the GUT theory. This is beyond the scope of this paper. Therefore, we will leave unspecified the field content above $M_{\mathrm{GUT}}$ and the mechanism responsible for GUT breaking down to the PS group.

On the other hand, at energies significantly below the GUT scale, there are good reasons to expect the effective theory as a (possibly supersymmetric) weakly interacting four-dimensional quantum field theory, where further symmetry breakings are realized by a conventional Higgs mechanism. We will take this as a working hypothesis. Without solid knowledge about the available PS multiplets below the GUT scale, we could allow for any set of group representations as the field content of the effective theory. Instead, we follow a simple phenomenological approach and restrict our analysis to a set of small representations. This set is chosen such that it is just sufficient to realize all possible chains of further symmetry breaking.

Below $M_{\mathrm{GUT}}$, we are thus dealing with a PS model which we specify in more detail. In the supersymmetric case, we list all the PS-symmetric supermultiplets that are contained in the spectrum. All such supermultiplets can interact and contribute to the running of the gauge couplings. The effective Lagrangian may contain all symmetric renormalizable interactions of these superfields. It will also contain, in general, non-renormalizable interactions of arbitrary dimension which are suppressed by powers of the relevant higher mass scales, i.e., GUT or Planck scales. While non-renormalizable terms, as it turns out, are not required for the symmetry-breaking chains that we discuss below, they can contribute significantly to flavor physics in the low-energy effective theory. We will not discuss non-renormalizable effects in this paper in any detail, so we do not attempt a complete description of flavor. The necessary inclusion of all kinds of subleading terms is beyond the scope of this paper.

For the supersymmetric PS model and the effective theories down to the SM, we state the following simplifying assumptions: 


\begin{tabular}{|c|ccc|}
\hline Field & $\left(\mathrm{SU}(4), \mathrm{SU}(2)_{L}, \mathrm{SU}(2)_{R}\right)$ & $\mathrm{SO}(10)$ & $E_{6}$ \\
\hline$\Sigma$ & $(\mathbf{1 5}, \mathbf{1}, \mathbf{1})$ & $\mathbf{4 5}$ & \\
$T_{L} \oplus T_{R}$ & $(\mathbf{1}, \mathbf{3}, \mathbf{1}) \oplus(\mathbf{1}, \mathbf{1}, \mathbf{3})$ & & $\mathbf{7 8}$ \\
$E$ & $(\mathbf{6}, \mathbf{2}, \mathbf{2})$ & $\mathbf{1 6}$ & \\
$\Phi_{L} \oplus \Phi_{R}$ & $(\mathbf{4}, \mathbf{2}, \mathbf{1}) \oplus(\overline{\mathbf{4}}, \mathbf{1}, \mathbf{2})$ & $\overline{\mathbf{1 6}}$ \\
$\bar{\Phi}_{L} \oplus \bar{\Phi}_{R}$ & $(\overline{\mathbf{4}}, \mathbf{2}, \mathbf{1}) \oplus(\mathbf{4}, \mathbf{1}, \mathbf{2})$ & $\mathbf{1}$ \\
$S_{78}$ & $(\mathbf{1}, \mathbf{1}, \mathbf{1})$ & $\mathbf{1 6}$ \\
$\Psi_{L} \oplus \Psi_{R}$ & $(\mathbf{4}, \mathbf{2}, \mathbf{1}) \oplus(\overline{\mathbf{4}}, \mathbf{1}, \mathbf{2})$ & \\
$h$ & $(\mathbf{1}, \mathbf{2}, \mathbf{2})$ & $\mathbf{1 0}$ \\
$F$ & $(\mathbf{6}, \mathbf{1}, \mathbf{1})$ & $\mathbf{1}$ \\
$S_{27}$ & $(\mathbf{1}, \mathbf{1}, \mathbf{1})$ & $\mathbf{2 7}$ \\
\hline
\end{tabular}

Table 1. Chiral superfield multiplets of the PS models considered in this paper. The fields are classified by their gauge-group quantum numbers; the discrete $Z_{\mathrm{LR}}$ symmetry renders the multiplets left-right symmetric and thus irreducible.

1. The model is effectively a perturbative quantum field theory in four dimension at all scales below $M_{\mathrm{GUT}}$.

2. The breaking of the PS symmetry and its subgroups is due to Higgs mechanisms that involve the superfields contained in the given PS spectrum.

3. SUSY is broken softly by terms in the TeV energy range.

4. The effective scale-dependent gauge couplings coincide at $M_{\mathrm{GUT}}$, consistent with a GUT symmetry that contains $\mathrm{SO}(10)$.

5. The chiral supermultiplets all fit in the lowest-lying $(\mathbf{1}, \mathbf{2 7}$, and $\mathbf{7 8})$ representations of $E_{6}$ or, equivalently, in $\mathbf{1}, \mathbf{1 0}, \mathbf{1 6}, \overline{\mathbf{1 6}}$, and 45 representations of $\mathrm{SO}(10)$.

The last point is the most important restriction that we impose on our models. (For a recent survey with different assumptions, see [9, 25]). As we will discuss below, this minimal field content can realize all possible chains of PS sub-symmetry breaking and addresses the phenomenological questions that we raised in the Introduction above. Simultaneously, a model with only small representations can easily be matched to a more ambitious theory of Planck-scale symmetry breaking. For instance, all multiplets that we consider, occur in the fundamental 248 representation of $E_{8}$ [37].

We now state explicitly the field spectrum that is consistent with our assumptions and display all possible irreducible PS multiplets in table 1. The matter fields of the MSSM are contained in $\Psi_{L / R}$ multiplets. There are three copies of this representation, each one including a right-handed neutrino superfield. The other supermultiplets are new. They 
contain the MSSM Higgs bidoublet, various new Higgs superfields, and extra "exotic" matter. Let us take a closer look at these superfields:

1. $h$ could directly qualify as the MSSM Higgs bi-doublet.

2. $\Sigma$ and $T_{L / R}$ are chiral multiplets in the adjoint representation. They have the same quantum numbers as the gauge fields of $\mathrm{SU}(4)_{C}$ and $\mathrm{SU}(2)_{L, R}$, respectively.

3. $\Phi_{L / R}$ and $\bar{\Phi}_{L / R}$ are extra multiplets with matter quantum numbers and their chargeconjugated images, respectively. Both must coexist in equal number, otherwise additional chiral matter generations would be present at the TeV scale. ${ }^{4}$

4. $E$ and $F$ contain only colored fields. Under $\mathrm{SU}(3)_{C}$ they decompose into triplets and anti-triplets, so they can be viewed as vector-like quarks and their scalar superpartners. Depending on their couplings, they may also behave as leptoquarks or diquarks. The $F$ multiplet may have both leptoquark and diquark couplings and thus can violate baryon number (cf. section 5.4). For the $E$ multiplet, this is not possible. Note that $\Sigma$ also provides vector-like quark and antiquark superfields, together with a color-octet and a color-singlet superfield. The $\Sigma$ couplings conserve baryon number.

5. The singlet fields $S_{78}$ and $S_{27}$ (and any further singlet fields that originate from $\mathrm{SO}(10)$ or $E_{6}$ singlets) can couple to any gauge-invariant quadratic polynomial.

Chiral superfields which are not copies of the above are excluded by our basic assumptions.

An explicit (i.e., PS invariant) mass term in the superpotential can be either present in the superpotential $a b$ initio, or generated by a singlet condensate. Without further dynamical assumptions, mass terms and mass thresholds are thus constrained only by matching conditions on the coupling parameters, and may exhibit a hierarchical pattern.

Some of the above mentioned multiplets contain an electroweak singlet and thus qualify as Higgs superfields for various steps of PS symmetry breaking, if the corresponding scalar component acquires a vacuum expectation value (vev). The associated vevs may be denoted as $\langle T(1,1,3)\rangle,\langle\Sigma(15,1,1)\rangle,\langle\Phi(4,1,2)\rangle$, and $\langle h(1,2,2)\rangle$, corresponding to the Higgs fields introduced below. They induce the following breaking patterns:

$$
\begin{gathered}
\mathrm{SU}(4)_{C} \stackrel{\langle\Sigma\rangle}{\longrightarrow} \mathrm{SU}(3)_{C} \times \mathrm{U}(1)_{B-L}, \\
\mathrm{SU}(2)_{R} \stackrel{\langle T\rangle}{\longrightarrow} \mathrm{U}(1)_{R}, \\
\mathrm{SU}(4)_{C} \times \mathrm{U}(1)_{R} \stackrel{\langle\Phi\rangle}{\longrightarrow} \mathrm{SU}(3)_{C} \times \mathrm{U}(1)_{Y}, \\
\mathrm{SU}(2)_{R} \times \mathrm{U}(1)_{B-L} \stackrel{\langle\Phi\rangle}{\longrightarrow} \mathrm{U}(1)_{Y}, \\
\mathrm{U}(1)_{R} \times \mathrm{U}(1)_{B-L} \stackrel{\langle\Phi\rangle}{\longrightarrow} \mathrm{U}(1)_{Y}, \\
\mathrm{SU}(2)_{L} \times \mathrm{U}(1)_{Y} \stackrel{\langle h\rangle}{\longrightarrow} \mathrm{U}(1)_{E} .
\end{gathered}
$$

\footnotetext{
${ }^{4} \mathrm{~A}$ fourth chiral generation is not finally excluded, but unlikely in view of present data.
} 


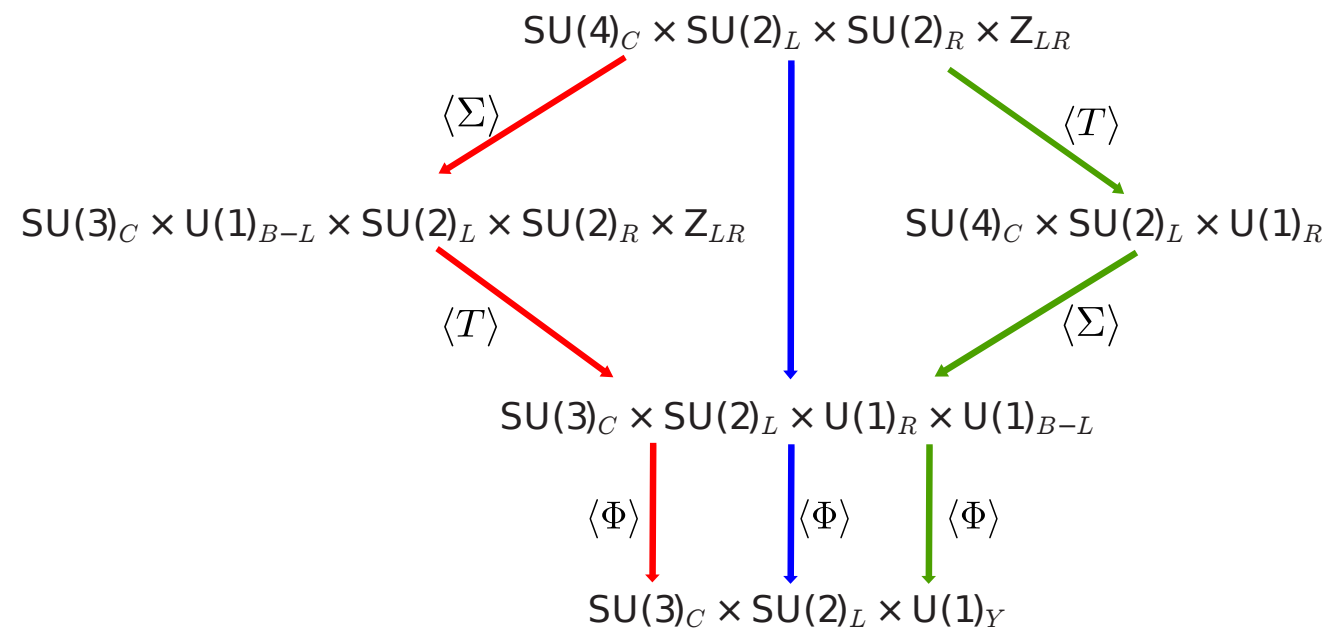

Figure 1. Graphical illustration of the different breaking paths depending on the different classes. Class B is shown in red, C in green, and D in blue.

If there is a hierarchy between the vevs, we observe a cascade of intermediate symmetries. Thus, we have the possibility for multi-step symmetry breaking. In the following, we distinguish six classes of PS models by their hierarchy patterns. We denote them as follows:

$\mathrm{A}:\langle\Phi\rangle \sim\langle T\rangle \sim\langle\Sigma\rangle$ (one scale)

B: $\langle\Phi\rangle \ll\langle T\rangle \ll\langle\Sigma\rangle$ (three scales),

$\mathrm{C}:\langle\Phi\rangle \ll\langle\Sigma\rangle \ll\langle T\rangle$ (three scales),

D: $\langle\Phi\rangle \ll\langle T\rangle \sim\langle\Sigma\rangle$ (two scales),

$\mathrm{E}:\langle\Phi\rangle \ll\langle\Sigma\rangle$ and $\langle T\rangle=0$ (two scales),

$\mathrm{F}:\langle\Phi\rangle \ll\langle T\rangle$ and $\langle\Sigma\rangle=0$ (two scales).

The symmetry breaking chains associated to these classes are shown in figure 1 (see also [28]).

The scale $\langle\Phi\rangle$ is always the lowest symmetry-breaking scale (above the electroweak scale), since this vev breaks all symmetries. The other vevs break the PS symmetry only partially and are only relevant if they lie above $\langle\Phi\rangle$. Nevertheless they are needed because we require the symmetry breaking to result from a renormalizable potential, which does not exist for $\Phi$ alone. Class A and D are limiting cases of the other and will not be discussed in greater detail.

The number of matter multiplets $\left(\Psi_{L / R}\right)$ is fixed to three. All other multiplets may appear in an arbitrary number of copies. We will specifically study scenarios where the multiplicity is either zero, one, or three. This already provides a wide range of possibilities for spectra and hierarchies and appears most natural in view of the generation pattern that is established for SM matter. 
Let us further categorize models, constructed along these lines, that we will study below. Each model type may fit into any of the above classes. It contains the three generations of MSSM matter superfields $\Psi_{L / R}$ together with the following extra superfields:

Type m (minimal model). Within a given class, the minimal model is the one with minimal field content that realizes the corresponding symmetry-breaking chain. In classes A to D, this model contains a single copy of each of the multiplets $\Phi, \Sigma$ and $T_{L / R}$. In classes $\mathrm{E}(\mathrm{F}), T_{L / R}(\Sigma)$ is omitted, respectively.

Type s (SO(10)-like model). In this model type, the multiplets can be composed to complete $\mathrm{SO}(10)$ representations. All multiplets listed in table 1 are present as a single copy.

Type e ( $\boldsymbol{E}_{\boldsymbol{6}}$-like model). In this type of model, all fields of table 1 are present. The multiplets $h, F$, and $S_{27}$ appear in three copies since they combine with the matter multiplets. For the other multiplets, we set the multiplicity to one.

Type g (generic model). Here we classify all models that do not qualify as either of the above three types. We will denote these models by their class and a unique number. The numbering scheme is described in appendix C.

\section{Higgs mechanism and supersymmetry}

Some of the superfields that we introduce beyond the MSSM matter fields, should act as Higgs fields and break the gauge symmetry, step by step, down to the SM gauge group. For each breaking step, we have to verify that the scalar potential has a local minimum for a non vanishing field configuration that breaks the gauge symmetry in the appropriate way. The value of the superpotential at this field configuration should be zero, so supersymmetry is maintained in the ground state.

To achieve this, we construct a generic renormalizable superpotential for the multiplets that we include in a specific model. Non-renormalizable terms could be added, but we will see that the various routes of gauge symmetry breaking result from renormalizable terms alone, so they can be ignored in a first approach. The specific models derive from a generic superpotential that includes all allowed superfields and their renormalizable interactions.

\subsection{Generic superpotential}

We start at the GUT scale with the most general renormalizable PS-invariant superpotential for the superfields shown in table 1 and impose parity $\left(Z_{L / R}\right)$ as a symmetry at this scale. The superpotential can be broken down in several parts which we organize in view of the model types $\mathrm{m}, \mathrm{s}$, and e as introduced above.

$$
W=W_{\Phi / \Sigma / T}+W_{\mathrm{h} / \mathrm{F}}+W_{\mathrm{E}}+W_{\mathrm{S}}+W_{\text {Yukawa }}
$$

The terms within $W_{\Phi / \Sigma / T}$ generate the Higgs potential for all steps in the staged Higgs mechanism. This superpotential consists only of those fields that are allowed to get a vev. 
Depending on the desired vev structure (case A to F), we may set some terms to zero, to obtain a minimal superpotential.

The superpotential $W_{h / F}$ is present in the models that contain the fields $h$ and $F$. Similarly, the field $E$ comes with the terms $W_{\mathrm{E}}$. The potential $W_{\mathrm{S}}$ contains all interactions of the singlets with the other Higgs fields, where in each term, $S$ indicates an arbitrary linear combination of all PS singlets present in the model.

If $h, F$ are present, there is the $W_{\text {Yukawa }}$ superpotential. This part, which is the only renormalizable superpotential involving (two) matter superfields, ${ }^{5}$ implicitly contains generation indices. Analogously, generation indices are implied for all superfields that occur in more than one copy. We note that $Y$ contains only symmetric Yukawa couplings that are universal across leptons, neutrinos, up- and down-type quarks.

$$
\begin{aligned}
W_{\Phi / \Sigma / T}= & -m_{\Phi}\left(\overline{\boldsymbol{\Phi}}_{L} \boldsymbol{\Phi}_{L}+\overline{\boldsymbol{\Phi}}_{R} \boldsymbol{\Phi}_{R}\right) \\
& -\frac{1}{2} m_{\Sigma} \boldsymbol{\Sigma}^{2}+\frac{1}{3} l_{\Sigma} \boldsymbol{\Sigma}^{3}+l_{\Sigma \Phi}\left(\overline{\boldsymbol{\Phi}}_{L} \boldsymbol{\Sigma} \boldsymbol{\Phi}_{L}+\overline{\boldsymbol{\Phi}}_{R} \boldsymbol{\Sigma} \boldsymbol{\Phi}_{R}\right) \\
& -\frac{1}{2} m_{T}\left(\boldsymbol{T}_{L}^{2}+\boldsymbol{T}_{R}^{2}\right)+l_{T \Phi}\left(\overline{\boldsymbol{\Phi}}_{L} \boldsymbol{T}_{L} \boldsymbol{\Phi}_{L}+\overline{\boldsymbol{\Phi}}_{R} \boldsymbol{T}_{R} \boldsymbol{\Phi}_{R}\right) \\
W_{\mathrm{h} / \mathrm{F}}= & -\frac{1}{2} m_{h} \boldsymbol{h}^{2}-\frac{1}{2} m_{F} \boldsymbol{F}^{2}+l_{h \Phi} \boldsymbol{h}\left(\boldsymbol{\Phi}_{L} \boldsymbol{\Phi}_{R}+\overline{\boldsymbol{\Phi}}_{R} \overline{\boldsymbol{\Phi}}_{L}\right) \\
& +l_{F \Phi} \boldsymbol{F}\left(\boldsymbol{\Phi}_{L} \boldsymbol{\Phi}_{L}+\boldsymbol{\Phi}_{R} \boldsymbol{\Phi}_{R}\right)+l_{F \bar{\Phi}} \boldsymbol{F}\left(\overline{\boldsymbol{\Phi}}_{L} \overline{\boldsymbol{\Phi}}_{L}+\overline{\boldsymbol{\Phi}}_{R} \overline{\boldsymbol{\Phi}}_{R}\right) \\
& +l_{\Sigma F} \boldsymbol{F} \boldsymbol{\Sigma} \boldsymbol{F}+l_{T h} \boldsymbol{h}\left(\boldsymbol{T}_{L}+\boldsymbol{T}_{R}\right) \boldsymbol{h} \\
W_{\mathrm{E}}= & -\frac{1}{2} m_{E} \boldsymbol{E}^{2}+l_{T E} \boldsymbol{E}\left(\boldsymbol{T}_{L}+\boldsymbol{T}_{R}\right) \boldsymbol{E} \\
& +l_{\Sigma E} \boldsymbol{E} \boldsymbol{\Sigma} \boldsymbol{E}+l_{F E h} \boldsymbol{F} \boldsymbol{E} \boldsymbol{h} \\
W_{\mathrm{S}}= & -\frac{1}{2} m_{S} \boldsymbol{S}^{2}+\frac{1}{3} l_{S} \boldsymbol{S}^{3} \\
& +s_{\Phi} \boldsymbol{S}\left(\overline{\boldsymbol{\Phi}}_{L} \boldsymbol{\Phi}_{L}+\overline{\boldsymbol{\Phi}}_{R} \boldsymbol{\Phi}_{R}\right)+s_{T} \boldsymbol{S}\left(\boldsymbol{T}_{L}^{2}+\boldsymbol{T}_{R}^{2}\right)+s_{\Sigma} \boldsymbol{S} \boldsymbol{\Sigma}^{2} \\
& +s_{h} \boldsymbol{S} \boldsymbol{h}^{2}+s_{F} \boldsymbol{S} \boldsymbol{F}^{2}+s_{E} \boldsymbol{S} \boldsymbol{E}^{2} \\
W_{\text {Yukawa }}= & Y \boldsymbol{\Psi}_{L} \boldsymbol{h} \boldsymbol{\Psi}_{R}+Y_{F} \boldsymbol{F}\left(\boldsymbol{\Psi}_{L} \boldsymbol{\Psi}_{L}+\boldsymbol{\Psi}_{R} \boldsymbol{\Psi}_{R}\right)
\end{aligned}
$$

\subsection{Higgs mechanism and supersymmetry}

For our supersymmetric models, we want to keep SUSY unbroken down to the TeV (MSSM) scale. To satisfy this constraint, we have to verify that all $F$ and $D$ terms vanish $[38,39]$ after inserting the vacuum expectation values of the fields: ${ }^{6}$

$$
\left.F_{i}\right|_{\mathrm{vev}}=0 \text { and }\left.\quad D_{a}\right|_{\mathrm{vev}}=0 .
$$

\footnotetext{
${ }^{5}$ Only $\Psi_{L}$ and $\Psi_{R}$ are considered as matter superfields, containing all fermions of the SM.

${ }^{6}$ We note that these conditions are trivially satisfied if all expectation values vanish. Without specifying further (possibly non-perturbative) dynamics, for a supersymmetric Higgs mechanism there is the alternative of no symmetry breaking, degenerate in energy. A complete theory should include a mechanism for supersymmetry breaking that lifts this degeneracy.
} 
We explicitly calculate the scalar potential for the generic superpotential shown in (4.1). This covers all models under consideration; for any specific model, we set the couplings to zero that are not involved.

The fields that transform under PS but contain a singlet under the SM gauge group, are $\Phi_{R}, \bar{\Phi}_{R}, \Sigma, T_{R}$, and the matter superfields $\Psi_{R}$. In particular, the $\mathrm{SU}(4)_{C}$ singlets in $\Phi_{R}$ and $\bar{\Phi}_{R}$ simultaneously break $\mathrm{SU}(2)_{R}$ and thus induce direct breaking PS $\rightarrow$ MSSM. However, there is no nontrivial renormalizable superpotential of $\Phi_{R}, \bar{\Phi}_{R}$ alone, so we include the $\Sigma$ and $T_{R}$ multiplets in the discussion. The vevs $\langle\Sigma\rangle$ and $\left\langle T_{R}\right\rangle$ are particularly relevant to the symmetry-breaking chain if they are significantly larger than $v_{\Phi}$, since they leave subgroups of PS intact (cf. figure 1). We introduce the following notation:

$$
\begin{aligned}
\left\langle\boldsymbol{\Phi}_{R}\right\rangle & =\Phi_{4,1,1}^{R}=v_{\Phi}, \\
\left\langle\overline{\boldsymbol{\Phi}}_{R}\right\rangle & =\bar{\Phi}_{4,1,1}^{R}=v_{\bar{\Phi}}, \\
\langle\boldsymbol{\Sigma}\rangle & =\Sigma_{15,1,1}=v_{\Sigma}, \\
\left\langle\boldsymbol{T}_{R}\right\rangle & =T_{1,1,3}=v_{T} .
\end{aligned}
$$

Here the subscript denotes the field component (or generator) that acquires the vev. These are all vevs we allow for in this paper.

For flavor physics, the vevs $v_{\Phi}$ and $v_{T}$ are of particular interest, because they break the discrete $Z_{\mathrm{LR}}$ symmetry. As long as the left-right symmetry is unbroken, there are no terms that distinguish right-handed up-type from down-type quarks. Therefore, the energy scale below which flavor mixing appears in the renormalizable part of the effective theory, is given by $v_{\Phi}$ or $v_{T}$. On the other hand, both $v_{\Sigma}$ and $v_{\Phi}$ separate quarks from leptons, so only in class-F models where $v_{\Sigma}=0$, we expect a direct relation between lepton-flavor and quark-flavor mixing.

The ground-state values of $\mathrm{D}$ terms are zero if and only if the vevs of mutually conjugate fields exist simultaneously and coincide in value. For $T$ and $\Sigma$ the D-terms automatically vanish since their generators are traceless. Therefore, we must have $\left\langle\Phi_{R}\right\rangle=\left\langle\bar{\Phi}_{R}\right\rangle$ :

$$
v_{\Phi} \equiv v_{\bar{\Phi}} .
$$

The vacuum expectation values of the $\mathrm{F}$ terms also have to vanish. Inserting the vevs, we obtain the necessary and sufficient conditions

$$
\begin{aligned}
& 0=F_{\Phi_{4}}=v_{\Phi}\left(m_{\Phi}-l_{\Sigma \Phi} v_{\Sigma}-l_{T \Phi} v_{T}\right), \\
& 0=F_{\Sigma_{15}}=v_{\Sigma}\left(m_{\Sigma}-l_{\Sigma} v_{\Sigma}\right)-l_{\Sigma \Phi} v_{\Phi}^{2}, \\
& 0=F_{T_{3}^{R}}=m_{T} v_{T}-l_{T \Phi} v_{\Phi}^{2} \\
& 0=F_{S}=s_{\Phi} v_{\Phi}^{2}+s_{\Sigma} v_{\Sigma}^{2}+s_{T} v_{T}^{2} .
\end{aligned}
$$


Solving for the mass parameters of $\Phi, T$ and $\Sigma$ as well as for one of the singlet couplings, we obtain

$$
\begin{aligned}
& m_{\Phi}=l_{\Sigma \Phi} v_{\Sigma}+l_{T \Phi} v_{T}, \\
& m_{T}=\frac{l_{T \Phi} v_{\Phi}^{2}}{v_{T}}, \\
& m_{\Sigma}=\frac{l_{\Sigma \Phi} v_{\Phi}^{2}}{v_{\Sigma}}+l_{\Sigma} v_{\Sigma}, \\
& s_{\Phi}=-\frac{s_{\Sigma} v_{\Sigma}^{2}+s_{T} v_{T}^{2}}{v_{\Phi}^{2}} .
\end{aligned}
$$

We have verified that these vev configurations also minimize the scalar potential while maintaining supersymmetry. Thus, depending on their mutual hierarchy, they realize the symmetry-breaking chains of the model classes A to D.

For the models classes $\mathrm{E}$ and $\mathrm{F}$, we assume a vanishing vev of $T$ or $\Sigma$, respectively. Nevertheless, PS symmetry is broken completely down to the MSSM gauge symmetry. A vanishing vev results if the corresponding multiplet does not couple to $\Phi_{R} / \bar{\Phi}_{R}$. This can be realized by either omitting the multiplet entirely, or by setting $l_{\Sigma \Phi}\left(l_{T \Phi}\right)$ to zero, respectively. Otherwise, SUSY would be broken.

As long as we stay in the regime of renormalizable superpotentials, it is not allowed to set $v_{\Phi}=0$, because in this case the solution of the minimization condition would be $v_{T} \equiv 0$, so $\mathrm{SU}(2)_{R}$ would remain intact. The reason is the absence of trilinear terms for $T_{R}$ in the renormalizable superpotential: $\mathrm{SU}(2)$ has no cubic invariant. In addition there would be no breaking of $\mathrm{U}(1)_{R} \times \mathrm{U}(1)_{B-L}$ down to hypercharge without $v_{\Phi}$.

\section{$5 \quad$ Spectra and phenomenology}

The various classes of models that we introduced above, lead to a great variety in the observable spectra. In the current work, we aim at a qualitative understanding, so we refrain from detailed numerical estimates or setting up benchmark models. Nevertheless, we observe a few characteristic patterns which can have interesting consequences for collider and flavor physics. We discuss these patterns and their phenomenological consequences below.

\subsection{Mass matrix}

Having verified that SUSY remains unbroken down to the TeV scale where soft-breaking terms appear, we consider the mass matrix of the scalars. We do not intend to derive quantitative results here, but just assign mass scales to the individual multiplets which depend on the hierarchy of the vevs. In table 2, we list the results for all fields and the interesting model classes B, C, E and F. The classes A and D are limiting cases. Therefore they are not listed separately.

We should keep in mind that this table refers only to the mass contributions that result from symmetry breaking. All superfields except for the matter multiplets, may carry an individual PS-symmetric bilinear superpotential term. These mass terms are 


\begin{tabular}{|cc|c|c|c|c|}
\hline Field & $\left(\mathrm{SU}(3)_{c}, \mathrm{SU}(2)_{L}\right)_{Y}$ & $\mathrm{~B}$ & $\mathrm{C}$ & $\mathrm{E}$ & $\mathrm{F}$ \\
\hline$\Sigma$ & $(\mathbf{8}, \mathbf{1})_{0}$ & $v_{\Sigma}$ & $v_{\Sigma}$ & $v_{\Sigma}$ & - \\
$E$ & $(\mathbf{3} / \overline{\mathbf{3}}, \mathbf{2})_{ \pm \frac{5}{6}}$ & - & - & - & - \\
$E$ & $(\mathbf{3} / \overline{\mathbf{3}}, \mathbf{2})_{ \pm \frac{1}{6}}$ & - & - & - & - \\
$\Phi_{L} / \bar{\Phi}_{L}$ & $(\mathbf{3} / \overline{\mathbf{3}}, \mathbf{2})_{ \pm \frac{1}{6}}$ & $v_{\Sigma}$ & $v_{T}$ & $v_{\Sigma}$ & $v_{T}$ \\
$\Phi_{R} / \bar{\Phi}_{R}$ & $(\mathbf{3} / \overline{\mathbf{3}}, \mathbf{1})_{ \pm \frac{2}{3}}$ & $v_{\Sigma}$ & $v_{\Sigma}$ & $v_{\Sigma}$ & $v_{\Phi}$ \\
$\Sigma$ & $(\mathbf{3} / \overline{\mathbf{3}}, \mathbf{1})_{ \pm \frac{2}{3}}$ & $v_{\Sigma}$ & $v_{\Sigma}$ & $v_{\Sigma}$ & - \\
$\Phi_{R} / \bar{\Phi}_{R}$ & $(\mathbf{3} / \overline{\mathbf{3}}, \mathbf{1})_{ \pm \frac{1}{3}}$ & $v_{\Sigma}$ & $v_{T}$ & $v_{\Sigma}$ & $v_{T}$ \\
$F$ & $(\mathbf{3} / \overline{\mathbf{3}}, \mathbf{1})_{ \pm \frac{1}{3}}$ & $\frac{v_{\Phi}^{2}}{v_{\Sigma}}$ & $\frac{v_{\Phi}^{2}}{v_{T}}$ & $\frac{v_{\Phi}^{2}}{v_{\Sigma}}$ & $\frac{v_{\Phi}^{2}}{v_{T}}$ \\
$T_{L}$ & $(\mathbf{1}, \mathbf{3})_{0}$ & $\frac{v_{\Phi}^{2}}{v_{T}}$ & $\frac{v_{\Phi}^{2}}{v_{T}}$ & - & $\frac{v_{\Phi}^{2}}{v_{T}}$ \\
$\Phi_{L} / \bar{\Phi}_{L}$ & $(\mathbf{1}, \mathbf{2})_{ \pm \frac{1}{2}}$ & $v_{T}$ & $v_{T}$ & $v_{\Phi}$ & $v_{T}$ \\
$h$ & $(\mathbf{1}, \mathbf{2})_{ \pm \frac{1}{2}}$ & $\frac{v_{\Phi}^{2}}{v_{T}}$ & $\frac{v_{\Phi}^{2}}{v_{T}}$ & $v_{\Phi}$ & $\frac{v_{\Phi}^{2}}{v_{T}}$ \\
$\Phi_{R} / \bar{\Phi}_{R}$ & $(\mathbf{1}, \mathbf{1})_{ \pm 1}$ & $v_{T}$ & $v_{T}$ & $v_{\Phi}$ & $v_{T}$ \\
$T_{R}$ & $(\mathbf{1}, \mathbf{1})_{ \pm 1}$ & $v_{T}$ & $v_{T}$ & - & $v_{T}$ \\
$T_{R}$ & $(\mathbf{1}, \mathbf{1})_{0}$ & $v_{\Phi}$ & $v_{T}$ & - & $v_{\Phi}$ \\
$\Sigma$ & $(\mathbf{1}, \mathbf{1})_{0}$ & $v_{\Sigma}$ & $v_{T}$ & $v_{\Sigma}$ & - \\
$\Phi_{R} / \bar{\Phi}_{R}$ & $(\mathbf{1}, \mathbf{1})_{0}$ & $v_{\Phi}$ & $v_{\Sigma}$ & $v_{\Phi}$ & $\frac{v_{\Phi}^{2}}{v_{T}}$ \\
$S_{27} / S_{78}$ & $(\mathbf{1}, \mathbf{1})_{0}$ & $\frac{v_{\Sigma}^{2}}{v_{T}}$ & $\frac{v_{\Sigma}^{3}}{v_{T}^{2}}$ & - & - \\
$S_{27} / S_{78}$ & $(\mathbf{1}, \mathbf{1})_{0}$ & $\frac{v_{\Sigma}^{2}}{v_{T}}$ & $\frac{v_{\Sigma}^{2}}{v_{T}^{2}}$ & $v_{\Phi}$ & $\frac{v_{\Phi}^{2}}{v_{T}}$ \\
\hline
\end{tabular}

Table 2. Mass hierarchy of the scalar fields in the different classes of the complete model. If non is shown, there is no contribution from the vev and their hierarchy is undefined. Class B: $v_{\Phi} \ll v_{T} \ll v_{\Sigma}$; class C: $v_{\Phi} \ll v_{\Sigma} \ll v_{T}$; class E: $v_{\Phi} \ll v_{\Sigma}, v_{T}=0$; class F: $v_{\Phi} \ll v_{T}, v_{\Sigma}=0$; the classes $\mathrm{A}$ and $\mathrm{D}$ are a limiting case of $\mathrm{B}$ and $\mathrm{C}$. Class $\mathrm{A}$ can be reached if one sets all vevs equal to a single vev $v$ and class $\mathrm{D}$ if one just sets $v_{\Sigma}=v_{T}=v$. The order is thus, that fields which mix are grouped together. Thus the mass eigenvalues are a linear combination of the listed fields. Massless components which are the Goldstone Bosons are explicitly not considered here.

completely arbitrary and, with supersymmetry, do not cause naturalness problems [40, 41]. Furthermore, a vev in any PS singlet field may contribute a similar term, again unrestricted by symmetries. Hence, by including a multiplet in the spectrum of the effective theory in a particular energy range, we implicitly assume that the sum of all mass terms for this field is either negligible compared to the energy, or fixed by the conditions that determine the vacuum expectation values.

On the other hand, in many models, not all of the fields $E, \Sigma$ and $T$ receive masses from PS and subsequent symmetry breaking, given only the renormalizable Lagrangian 
terms above. For these fields, either the bilinear mass term or effective masses induced by higher-dimensional operators play an important role and must be included as independent parameters. From a phenomenological viewpoint, we are mainly interested in the lowest possible mass for each of those fields. We discuss this issue in section 7.1.

While some multiplets acquire masses proportional to either one of the symmetrybreaking scales $v_{T}, v_{\Sigma}, v_{\Phi}$, there are various cases where the mass becomes of order $v_{\Phi}^{2} / v_{T}$ or $v_{\Phi}^{2} / v_{\Sigma}$, which can be significantly smaller than $v_{\Phi}$ if there is a hierarchy between the vevs. In other words, there is an extra see-saw effect, unrelated to the well-known neutrino see-saw $[27,42,43]$. We denote this induced mass scale by $M_{\text {IND. }}$ It is located below the scale where PS is completely broken down to the MSSM symmetry group. A generic expression is

$$
M_{\mathrm{IND}} \sim \frac{v_{\Phi}^{2}}{v_{\Sigma}+v_{T}} .
$$

Depending on the model class, some of the field multiplets $F, h$, or $T_{L}$ become associated with $M_{\mathrm{IND}}{ }^{7}$ (cf. table 2). We thus get "light" supermultiplets consisting of scalars and fermions, which may be colored, charged, or neutral, and acquire a mass that does not coincide with either of the symmetry-breaking scales. If the hierarchy between the vevs is strong, $M_{\text {IND }}$ may be sufficiently low to become relevant for collider phenomenology. In model classes B, C, and F, it provides a $\mu$ term for $h$ and may thus be related to electroweak symmetry breaking. In any case, the threshold $M_{\text {IND }}$ must be taken into account in the renormalization-group running of the gauge couplings.

\subsection{Goldstone bosons}

Not all of the scalar fields are physical: since the broken symmetries are gauged, nine of the scalar fields are Goldstone bosons that provide the longitudinal modes of the PS gauge bosons that are integrated out in the breaking down to the MSSM. Six of them come from $\mathrm{SU}(4)_{C} \rightarrow \mathrm{SU}(3)_{C}$ (5.2a), (5.2b), and two additional ones implement $\mathrm{SU}(2)_{R} \rightarrow \mathrm{U}(1)_{R}$ (5.2c), (5.2d). The last one comes from the breaking of the $\mathrm{U}(1)$ subgroups $\mathrm{U}(1)_{B-L} \otimes \mathrm{U}(1)_{R} \rightarrow \mathrm{U}(1)_{Y}$ (5.2e). We identify these Goldstone bosons as

$$
\begin{aligned}
G B_{1,2,3} & =-\mathrm{i} \frac{\sqrt{3}}{2} \frac{v_{\Phi}}{v_{\Sigma}} \boldsymbol{\Phi}_{3}^{R}-\mathrm{i} \frac{\sqrt{3}}{2} \frac{v_{\Phi}}{v_{\Sigma}} \overline{\boldsymbol{\Phi}}_{3}^{*}+\boldsymbol{\Sigma}_{3}+\boldsymbol{\Sigma}_{3}^{*}, \\
G B_{4,5,6} & =\mathrm{i} \frac{\sqrt{3}}{2} \frac{v_{\Phi}}{v_{\Sigma}} \boldsymbol{\Phi}^{*} \frac{R}{3}+\mathrm{i} \frac{\sqrt{3}}{2} \frac{v_{\Phi}}{v_{\Sigma}} \overline{\boldsymbol{\Phi}}_{3}^{R}+\boldsymbol{\Sigma}_{\overline{3}}+\boldsymbol{\Sigma}^{*}{ }_{3}, \\
G B_{7} & =\boldsymbol{T}_{1_{1}}^{R}+\boldsymbol{T}_{1_{1}}^{* R}-\frac{\mathrm{i}}{\sqrt{2}} \frac{v_{\Phi}}{v_{T}} \overline{\boldsymbol{\Phi}}_{1_{1}}^{R}-\frac{\mathrm{i}}{\sqrt{2}} \frac{v_{\Phi}}{v_{T}} \boldsymbol{\Phi}_{1_{1}}^{* R}, \\
G B_{8} & =\boldsymbol{T}_{1_{-1}}^{R}+\boldsymbol{T}_{1_{-1}}^{* R}+\frac{\mathrm{i}}{\sqrt{2}} \frac{v_{\Phi}}{v_{T}} \boldsymbol{\Phi}_{1_{-1}}^{R}+\frac{\mathrm{i}}{\sqrt{2}} \frac{v_{\Phi}}{v_{T}} \overline{\boldsymbol{\Phi}}_{1_{-1}}^{R}, \\
G B_{9} & =\operatorname{Im}\left(\boldsymbol{\Phi}_{1_{0}}^{R}\right)-\operatorname{Im}\left(\overline{\boldsymbol{\Phi}}_{1_{0}}^{R}\right) .
\end{aligned}
$$

Here 3 and $\overline{3}$ are the $(\mathbf{3}, \mathbf{1})_{\frac{2}{3}}$ and $(\overline{\mathbf{3}}, \mathbf{1})_{\frac{2}{3}}$ components of the Higgs fields $\boldsymbol{\Phi}_{R}, \overline{\mathbf{\Phi}}_{R}$ and $\boldsymbol{\Sigma}$, respectively. For vanishing vevs, the corresponding fields do not mix into the GBs. Thus, if $v_{\Sigma}=0\left(v_{T}=0\right)$, the Goldstone bosons $G B_{1-6}\left(G B_{7 / 8}\right)$ are only mixtures of $\Phi$.

\footnotetext{
${ }^{7}$ In class $\mathrm{F}$, this applies also to the singlet part of $T_{R}$.
} 


\subsection{MSSM Higgs}

Apart from the matter fields and Goldstone bosons, the chiral superfield spectrum must provide the Higgs bi-doublet of the MSSM that is responsible for electroweak symmetry breaking. Above the TeV scale, this multiplet should appear as effectively massless; mass terms (which together set the EWSB scale) are provided by soft-SUSY breaking parameters and the $\mu$ term which mixes both doublets. We note that the $\mu$ term may result from the vev of one of the electroweak singlets in the model, i.e., the model may implement a NMSSM-type solution for the $\mu$ problem [44, 45].

The obvious candidate for the MSSM Higgs bi-doublet is the superfield $h$. From table 2 we read off that the PS-breaking contribution to the $h$ mass is see-saw suppressed in models with $v_{T} \neq 0$ (B, C, D and F). In these models, the electroweak hierarchy is generated, at least partly, by a high-energy hierarchy in the PS symmetry-breaking chain.

However, the $\Phi_{L}$ multiplets provide further Higgs candidates. In particular, while the right-handed doublets in $\Phi_{R}$ serve as Goldstone bosons for the $\mathrm{SU}(2)_{R}$ breaking and are thus unphysical, the mirror images in $\Phi_{L}$ are to be considered as physical scalars (above the EWSB scale). Above the L-R breaking scale, these fields are protected against mass terms, since all contributions to the mass are absorbed in the minimization conditions. ${ }^{8}$ Therefore they can only acquire nonzero mass due to mixing effects proportional to $l_{h \Phi}$ and $l_{T \Phi}$ breaking LR-symmetry. With respect to the SM gauge symmetry, they have the same quantum numbers as $h$, and will be called $h_{\Phi}$ in the following.

In the limit $v_{\Phi} \ll v_{T}$, the mixing effect which provides a mass for $h_{\Phi}$ becomes negligible, while there may still be an large contribution to the $h$ mass. For the $h / h_{\Phi}$ system, we obtain a mass matrix with an approximate eigenvalue structure

$$
\begin{aligned}
\mu & \approx m_{h}+\frac{l_{h \Phi}^{2}}{l_{T \Phi}} \frac{v_{\Phi}^{2}}{v_{T}}, \\
m_{h_{\Phi}}^{\prime} & \approx l_{T \Phi} v_{T},
\end{aligned}
$$

where we allow for $m_{h}$ as an independent $\mu$ term, not directly related to PS breaking. Both mass terms may be as low as the $\mathrm{TeV}$ scale where soft-breaking terms come into play. In other words, the MSSM Higgs bi-doublet (in particular, the Higgs boson that has been observed) may belong to either $h$ or $h_{\Phi}$, or be a mixture of both.

If $v_{T}$ vanishes (class $\mathrm{E}$ ), the situation becomes more complicated. Now, both masses get a contribution of the order $l_{h \Phi} v_{\Phi}$. For $m_{h}=0$, both mass eigenvalues are degenerate. Conversely, if the mixing between $h$ and $h_{\Phi}$ vanishes, they are maximally split $\left(m_{h}, 0\right)$. For small mixing $\left(l_{h \Phi} m_{h} \ll v_{\Phi}\right)$, we get an additional see-saw effect and a factor $l_{h \Phi}^{2} v_{\Phi}^{2} / m_{h}$ for the smaller eigenvalue, which generates the effective $\mu$ term.

In short, in various classes of PS models, the MSSM Higgs bi-doublet may be naturally light, and it could actually originate from the $\Phi_{L}$ superfields.

\footnotetext{
${ }^{8}$ The mass of $\Phi_{R}$ is fixed since it is proportional to the vev which we keep fixed.
} 


\subsection{The $F$ multiplet}

The mass of the multiplet $F$ is generically see-saw suppressed and thus comparatively light. This appears as a common feature of all model classes with more than one scale. It can couple to matter via $W_{\text {Yukawa }}$. Since $F$ is a $\mathrm{SU}(4)_{C}$ antisymmetric tensor, the possible Yukawa couplings provide both diquark and leptoquark couplings, explicitly breaking baryon number in the low-energy theory. In fact, $F$ is the analog of the colored Higgs field which in SU(5) GUT models induces rapid proton decay unless it is very heavy.

However, in PS models the Yukawa matrices $Y_{F}$ and $Y$ are not related, so there is no doublet-triplet splitting problem [21, 22]. Hence, by omitting the coupling of $F$ to $\Psi$, proton decay is excluded in the presence of all gauge symmetries. This can be achieved, for instance, by a flavor symmetry or by an appropriate discrete quantum number.

If the $F$ multiplet is sufficiently light, it may provide detectable new particles at colliders. Without the $Y_{F}$ Yukawa coupling, there is no immediate decay to MSSM matter fields, but other terms in the Lagrangian provide indirect decay channels. In this situation, the particles (color-triplet scalars and fermion superpartners) may become rather narrow as resonances.

\subsection{SM singlets}

The most complicated mass matrix belongs to the electroweak singlets that are contained in the various PS multiplets. Even if we do not consider PS singlets, there are still five electroweak singlets which can mix non-trivially. In the general case, their masses cannot be calculated in closed analytical form. To get a handle on these particles, we computed the dependence on the different scales numerically. Given one of the scale hierarchy patterns introduced above, we find additional scales and new hierarchy patterns which may have interesting consequences for flavor and Higgs physics. However, singlets do not contribute to the running of the gauge couplings at leading-logarithmic level, so we do not attempt a detailed discussion of the singlet sector in the present work.

\subsection{Matter couplings}

The renormalizable superpotential contains terms that couple $h$ to matter $\Psi$, so matter fields can get masses via the $h$ vev, after electroweak symmetry breaking. However, there is no reason for flavor physics to originate solely from the renormalizable superpotential. In particular, if $h_{\Phi}$ turns out to be the MSSM Higgs, there are no contributions from this superpotential at all.

Instead, we expect significant and non-symmetric contributions to masses and mixing from non-renormalizable terms that induce effective Yukawa couplings at one of the symmetry-breaking scales. The generated terms are proportional to powers of the various vevs in the model, and suppressed by masses of heavier particles, by $M_{\mathrm{GUT}}$, or $M_{\text {Planck }}$. There is ample space for flavor hierarchy in the resulting coefficients. For instance, if we consider dimension-four terms in the superpotential, we identify the following interactions 
that can affect matter-Higgs Yukawa couplings in the low-energy effective theory,

$$
\begin{aligned}
W_{\text {Yukawa }}^{\mathrm{NLO}}= & \frac{Y_{h \Sigma}}{\Lambda} \boldsymbol{\Psi}_{L} \boldsymbol{h} \boldsymbol{\Sigma} \boldsymbol{\Psi}_{R}+\frac{Y_{\Phi}}{\Lambda} \boldsymbol{\Psi}_{L}\left(\boldsymbol{\Phi}_{L} \boldsymbol{\Phi}_{R}+\overline{\boldsymbol{\Phi}}_{L} \overline{\boldsymbol{\Phi}}_{R}\right) \boldsymbol{\Psi}_{R} \\
& +\frac{Y_{E F}}{\Lambda} \boldsymbol{\Psi}_{L} \boldsymbol{E} \boldsymbol{F} \boldsymbol{\Psi}_{R} .
\end{aligned}
$$

There are additional couplings to gauge singlets, which may also be flavored, so overall there is great freedom in assigning masses and generating hierarchies in the mass and mixing parameters.

\subsection{Neutrino mass}

As a left-right-symmetric extension of the SM, PS models contain right-handed neutrinos and allow for a Dirac neutrino mass term. Furthermore, the extra fields that are present in our setup can induce any of the three neutrino see-saw mechanisms for mass generation $[46,47]$. The fields $\Phi_{R}$ and $T_{R}$ provide singlets with a vev that can couple to right-handed neutrinos, generating a Majorana mass term proportional to $v_{\Phi}$ and $v_{T}$, respectively. Combined with the Dirac mass, this results in a type I see-saw. The field $T_{L}$ contains $\mathrm{SU}(2)_{L}$-triplet scalars and fermions and may thus induce a type-II or type-III see-saw mechanism.

\section{Unification conditions}

Within the framework of PS model classes that we have defined in the previous sections, we now impose unification conditions on the gauge couplings. As stated above, we require complete unification for all gauge groups to a GUT symmetry that contains $\mathrm{SO}(10)$. The unification scale $M_{\mathrm{GUT}}$ where this should happen is not fixed but depends on the spectrum. At each threshold below this where the spectrum changes, i.e., particles are integrated out, we state the appropriate matching conditions.

We work only to leading-logarithmic level, where non-abelian running gauge couplings are continuous in energy, and matching conditions depend only on the spectra. ${ }^{9}$ Abelian gauge couplings do exhibit discontinuous behavior as an artifact of differing normalization conventions in different effective theories.

The leading-logarithmic running of a gauge couplings between fixed scales $\mu_{1}$ to $\mu_{2}$ is given by

$$
\frac{1}{\alpha_{i}\left(\mu_{2}\right)}=\frac{1}{\alpha_{i}\left(\mu_{1}\right)}-\frac{b_{i}}{2 \pi} \ln \left(\frac{\mu_{2}}{\mu_{1}}\right) .
$$

Here, the $b_{i}$ are group theoretical factors that can be calculated from the representations of the particles [48].

\footnotetext{
${ }^{9}$ At next-to-leading order, the superpotential parameters enter the running. However, given the great freedom in choosing a model in the first place, there is little to be gained from including such effects in our framework.
} 
Inserting the intermediate mass scales (case B for definiteness) as discussed above, the complete running is a sum of multiple terms,

$$
\begin{aligned}
\frac{1}{\left.\alpha_{i}\left(M_{\mathrm{GUT}}\right)\right)}= & \frac{1}{\alpha_{i}\left(M_{Z}\right)}-\frac{b_{i}^{(1)}}{2 \pi} \ln \left(\frac{M_{\mathrm{SUSY}}}{M_{Z}}\right)-\frac{b_{i}^{(2)}}{2 \pi} \ln \left(\frac{M_{\mathrm{IND}}}{M_{\mathrm{SUSY}}}\right)-\frac{b_{i}^{(3)}}{2 \pi} \ln \left(\frac{v_{\Phi}}{M_{\mathrm{IND}}}\right) \\
& -\frac{b_{i}^{(4)}}{2 \pi} \ln \left(\frac{v_{T}}{v_{\Phi}}\right)-\frac{b_{i}^{(5)}}{2 \pi} \ln \left(\frac{v_{\Sigma}}{v_{T}}\right)-\frac{b_{i}^{(6)}}{2 \pi} \ln \left(\frac{M_{\mathrm{GUT}}}{v_{\Sigma}}\right)
\end{aligned}
$$

where $M_{\text {IND }}$ denotes the additional see-saw induces scale introduced in (5.1). Since this scale depends on the numerical values of superpotential parameters, which we do not determine, we treat this as a free parameter. Distinguishing different model classes with their corresponding hierarchy patterns, we have to appropriately adapt the ordering of scales and the definition of the $b_{i}^{(n)}$.

The calculation of the coefficients in this formula is straightforward and can be found in appendix A. For simplicity, we always assume that the listed scales exhaust the available hierarchies, and no further hierarchies from couplings become relevant here. Thus, we assume all additional scalar fields to be integrated out at their "natural" mass scale, which is determined by the considerations in the previous section.

In passing, we note that the calculation is actually independent from a supersymmetry assumption. The supersymmetric and non-supersymmetric frameworks differ only in the form of the $\beta$-function (cf. (A.5), (A.6)).

Regarding U(1) couplings with their normalization ambiguity, we have to explicitly consider the unification condition for

$$
\mathrm{U}(1)_{R} \otimes \mathrm{U}(1)_{B-L} \longrightarrow \mathrm{U}(1)_{\mathrm{Y}}
$$

To define the hypercharge coupling strength, we explicitly calculate the unbroken direction and identify the charges of the larger groups. This results in a relation between the group generators and therefore between charges and couplings. We obtain ${ }^{10}$

$$
\begin{aligned}
Y & =\frac{B-L}{2}+T_{3}^{R}, \\
\alpha_{Y}^{-1}\left(v_{\Phi}\right) & =\frac{2}{3} \alpha_{B-L}^{-1}\left(v_{\Phi}\right)+\alpha_{R}^{-1}\left(v_{\Phi}\right) .
\end{aligned}
$$

For the non-abelian symmetry breaking steps, the unification conditions just depend on the breaking pattern, i.e.,

$$
\begin{aligned}
\mathrm{GUT} & \longrightarrow \mathrm{SU}(4)_{C} \otimes \mathrm{SU}(2)_{L} \otimes \mathrm{SU}(2)_{R} \\
\mathrm{SU}(4)_{C} & \longrightarrow \mathrm{SU}(3)_{C} \otimes \mathrm{U}(1)_{B-L}, \\
\mathrm{SU}(2)_{R} & \longrightarrow \mathrm{U}(1)_{R}
\end{aligned}
$$

\footnotetext{
${ }^{10}$ We do not rescale $\mathrm{U}(1)$ in order to match the $\mathrm{SU}(5)$ normalization, as is often done in the literature.
} 
where the GUT group contains $\mathrm{SO}(10)$. These three breaking patterns result in the matching conditions ${ }^{11}$

$$
\begin{aligned}
\alpha_{4}^{-1}\left(M_{\mathrm{GUT}}\right) & =\alpha_{L}^{-1}\left(M_{\mathrm{GUT}}\right)=\alpha_{R}^{-1}\left(M_{\mathrm{GUT}}\right) \equiv \alpha_{\mathrm{GUT}}^{-1}\left(M_{\mathrm{GUT}}\right), \\
\alpha_{3}^{-1}\left(v_{\Sigma}\right) & =\alpha_{B-L}^{-1}\left(v_{\Sigma}\right) \equiv \alpha_{4}^{-1}\left(v_{\Sigma}\right) \\
\alpha_{\mathrm{U}(1)_{R}}^{-1}\left(v_{T}\right) & =\alpha_{R}^{-1}\left(v_{T}\right)=\alpha_{L}^{-1}\left(v_{T}\right)
\end{aligned}
$$

respectively.

In addition to the unification and matching conditions, we have the additional constraint that the mass scales are properly ordered. For instance, for class B we have:

$$
M_{\mathrm{SUSY}} \leq M_{\mathrm{IND}} \leq v_{\Phi} \leq v_{T} \leq v_{\Sigma} \leq M_{\mathrm{GUT}} \lesssim 10^{19} \mathrm{GeV}
$$

Furthermore, the coupling strengths $\alpha_{i}$ have to be sufficiently small and positive at all mass scales, so we do not leave the perturbative regime.

Counting the number of conditions and free parameters (scales), we observe that the models are still under-constrained. Hence, we can derive constraints for the mass scales and exclude particular models, but not fix all scales completely. Nevertheless, imposing unification does restrict the model parameter space significantly, as we can show in the following sections.

\section{Supersymmetric Pati-Salam models}

In this section, we study the supersymmetric models that are consistent with our set of assumptions. We scan over all models by varying the number of superfield generations $(0,1,3)$ that are present in each effective theory (i.e., between the various symmetry breaking scales), independently for each gauge multiplet.

We force the masses of all superfields that are not part of the low-energy (MSSM) spectrum, to coincide with one of the thresholds $M_{\mathrm{IND}}, v_{T}, v_{\Phi}$, etc., as explained in section 5 . In any case, order-one prefactors in the mass terms would only enter logarithmically in the gauge-coupling unification (6.2) and matching conditions (GCU) (6.4b), (6.6), (6.7). This is a minor uncertainty. We should note, however, that additional coupling hierarchies, as they exist in the flavor sector of the known matter particles, are also possible and lead to a wider range of possibilities which we do not investigate further.

With these conventions understood, the model scan will be exhaustive, since we vary just discrete labels. In total, there are 1078 distinct configurations. Since we now are dealing with mass scales rather then vev structures or specific mass eigenvalues, we change our notation from vevs to mass scales (see appendix B).

For each model, we analytically calculate the numerical values of mass scales for which the GCU conditions stated in the previous section can be fulfilled. Models where no solution is possible are not considered further. For the remaining models, we obtain model-specific relations between the mass scales. As a result, we can express those scales as functions

\footnotetext{
${ }^{11}$ In case one of the breaking steps is absent, the corresponding conditions apply at the next step below.
} 
of one or two independent mass parameters that we may choose as input. Varying those within the possible range, we obtain allowed ranges for all mass scales, within each model separately.

For all numerical results, we fix the common soft SUSY-breaking scale at $M_{\mathrm{SUSY}}=$ $2.5 \mathrm{TeV} .^{12}$

\subsection{Extra mass parameters}

As discussed in the previous section, in some model classes the fields $E, \Sigma$ and $T$ do not necessarily obtain a mass term from symmetry breaking. Thus, their masses must be treated as extra free parameters. To get a handle on these scales, we considered all possibilities for assigning the mass scales of these superfields to the other mass scales in our framework, while keeping the GCU conditions. As a result, we can exclude the possibility that these extra scales are at the lower end of the spectrum.

More specifically, in all model classes we find a lower bound for the colored $E$ multiplet of about $m_{E} \gtrsim 10^{8} \mathrm{GeV}$. Similar results apply to $\Sigma$ and $T$, if we do not consider lowering the GUT scale below about $10^{16} \mathrm{GeV}$. In other words, the proton stability constraint which limits the GUT scale, suggests that these fields have rather large masses. For the further scan over models, we fix their mass scales, whenever they are not determined by the vevs, at $M_{\mathrm{PS}}$.

Any model has to provide a candidate for the electroweak Higgs boson. This excludes a large invariant mass for the corresponding PS multiplet. We therefore do not include an explicit mass term for the $h$ superfield. We have checked to what extend $h_{\Phi}$ may serve as the low energy MSSM-like Higgs. This is possible, but only for class E. Thus, we must include at least one generation of $h$ in the classes B, C and F. This reduces our scan to 828 configurations.

\subsection{General overview}

Before we discuss the various classes and types of models in more detail, we summarize generic features and specific observations that we can extract from the study of all 828 supersymmetric models.

Roughly one half of all models are capable of GCU. Except for class E, all such configurations exhibit a unification scale $M_{\mathrm{GUT}}>10^{16} \mathrm{GeV}$ and are thus favored by the non-observation of proton decay. In class $\mathrm{E}$ this is true for half of them.

In contrast to the classes $\mathrm{C}, \mathrm{E}$ and $\mathrm{F}$, the allowed ranges in class $\mathrm{B}$ are rather constrained, so in this class, models can be fixed in a semi-quantitative way.

We now take a look at the low energy spectrum of the different classes. These can be extra color triplets $(F)$ or $\mathrm{SU}(2)_{L}$ triplets $\left(T_{L}\right)$. In particular, we are interested in models where the lowest new threshold $M_{\mathrm{IND}}$ is already in the TeV-range, so new particles are potentially accessible at colliders, while full unification occurs near the Planck scale. 114 models satisfy these conditions. 72 of them are categorized as class E and as such contain

\footnotetext{
${ }^{12}$ We also considered a lower SUSY-breaking scale of $M_{\mathrm{SUSY}}=250 \mathrm{GeV}$ which is disfavored by LHC data; it turns out that the unification conditions are generically easier to satisfy for the larger value of the soft SUSY-breaking scale.
} 


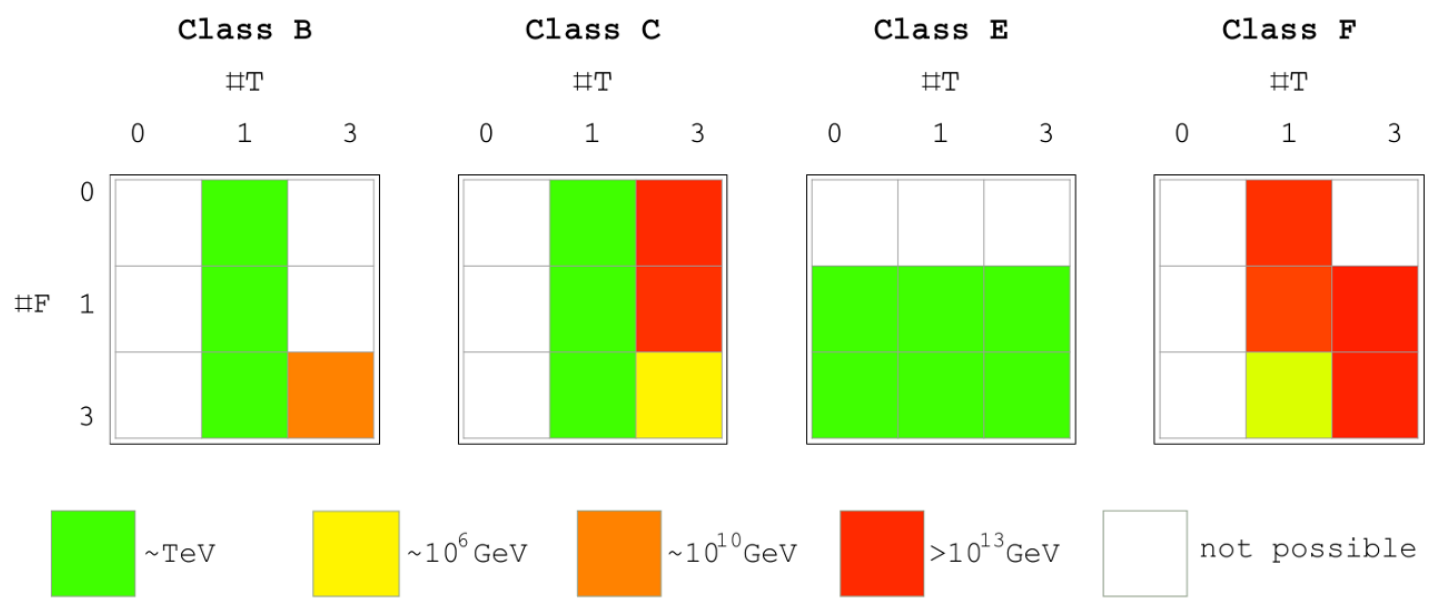

Figure 2. Graphical illustration of the lowest new mass scale, dependent on the multiplicity in the low-energy spectrum. We vary the number of low-lying $\mathrm{SU}(2)$ triplets $T$ ( $x$ axis) and low-lying color triplets $F$ ( $y$ axis), independently. The colors indicate the lowest mass scale, ranging from green (SUSY scale) to red (Planck-scale). White6 squares correspond to configurations not leading to GCU or which are inconsistent with the class definitions.

only light color triplets. 34 of them are categorized as class C. In class B, only a few models fulfill this condition, none in class F.

One key feature of our configurations is that we allow for up to six light $\mathrm{SU}(2)_{L}$ scalar bidoublets $h$ and $h_{\Phi}$ (three each). Most configurations with more than one fall in class E. But also in class $\mathrm{C}$ there is a handful of configurations. In class $\mathrm{E}$ most successful configurations have more than one light bidoublet. This is because $h$ and $h_{\Phi}$ are taken as degenerate in mass. A more detailed discussion will follow when we look explicitly at class E.

In class $\mathrm{E}$ the LR-breaking scale is allowed to be rather light. We find 120 distinct models with $M_{\mathrm{LR}}<10^{5} \mathrm{GeV}$. Also in class $\mathrm{C}$ we find some configurations.

As a generic observation, $\mathrm{SU}(2)$ triplets $T$ rarely get low mass, and, if present in the intermediate range, tend to be associated with lower GUT scales. One exception is class F with three generations of light triplets $F$. Here, light $T$ are realized for a GUT scale near $M_{\text {Planck }}$. Light color triplets $F$, i.e., extra quarks and their superpartners, are more common. Actually, in class E they are allowed over a large mass range for the GUT scale. In classes A to D, we may have color triplets around some $100 \mathrm{TeV}$, as long as there is only one generation of light $\mathrm{SU}(2)$ triplets. In class $\mathrm{F}$, the fields $T$ and $F$ are generically more heavy (cf. section 7.4).

We illustrate these results in figure 2. The figure displays a considerable fraction of models where new matter is possible at the lowest scales (green squares), so we should be prepared to observe exotic particles, or at least their trace in precision observables, at collider experiments.

Another observable of interest is the preferred mass range for right-handed neutrinos. In our setup, the Majorana mass parameter should be of the order $\langle\Phi\rangle$ where all symmetries 


\begin{tabular}{|l|c|c|c|c|c|}
\hline & class B & class C & class E & class F & $\sum$ \\
\hline scanned & 144 & 144 & 324 & 216 & 828 \\
GCU & 18 & 57 & 254 & 29 & 358 \\
$M_{\mathrm{GUT}}>10^{16} \mathrm{GeV}$ & 18 & 57 & 131 & 29 & 235 \\
$M_{\mathrm{IND}}<10 \mathrm{TeV}$ and $M_{\mathrm{GUT}}>10^{16} \mathrm{GeV}$ & 8 & 34 & 72 & 0 & 114 \\
$M_{\mathrm{LR}}<100 \mathrm{TeV}$ & 1 & 11 & 108 & 0 & 120 \\
$10^{12} \mathrm{GeV}<M_{N_{R}}<10^{14} \mathrm{GeV}$ & 16 & 42 & 123 & 3 & 184 \\
$M_{\mathrm{IND}} \in[0.1,10] \frac{v_{\Phi}^{2}}{v_{\Sigma}+v_{T}}$ & 14 & 20 & 203 & 26 & 263 \\
\hline
\end{tabular}

Table 3. Number of configurations full filling certain conditions.

that protect this term are broken. Scanning all configurations with respect to this scale, we find 184 with $10^{12} \mathrm{GeV} \lesssim M_{N_{R}} \lesssim 10^{14} \mathrm{GeV}$. Actually, in classes $\mathrm{B}$, C, three-quarter and in class $\mathrm{E}$ still half of all successful models fall in this category. Only in class $\mathrm{F}$, this scale is typically higher; only $10 \%$ of the models result in a value in this range.

We also find that a neutrino mass scale in this range is associated with at most one generation of $h$ fields. As an interesting non-standard scenario, hinting at $E_{6}$ grand unification, three light $h$ generations are also possible, but accompanied by heavier right-handed neutrinos. In classes B and C, we find a few successful models with three $h$ generations, and none in class F. However, there is quite some space for this scenario in class E.

So far, we treated $M_{\text {IND }}$ as a free parameter. To obtain more specific predictions, we imposed the restriction $M_{\mathrm{IND}} \in[0.1,10] \frac{v_{\Phi}^{2}}{v_{\Sigma}+v_{T}}$. While this does not significantly reduce the number of allowed models, it drastically reduces the configurations with $\mathrm{TeV}$-scale new particles. Most of these models still allowing TeV-scale new particles belong to class E. In this class, one quarter of all models allows for colored triplets below about $10 \mathrm{TeV}$.

In some models, the GCU constraints pin down all scales to a narrow range. The most obvious case is standard $\mathrm{SO}(10)$ coupling unification at the GUT scale, i.e., all vevs are of the same order of magnitude and located at $M_{\mathrm{GUT}}$. Clearly, this well-studied model is contained in our scan as a limiting case. We reproduce the observation that for this case, the only light multiplet is the MSSM Higgs $h$. However, we also find a few models where scales are essentially fixed, but the spectrum and unification pattern is different. Those are all classified as class E. More generic statistics can be read off table 3.

\subsection{Class E}

From the overview above we can conclude that class E contains the largest set of models with phenomenologically interesting features. In this class, the ordering of new thresholds is, in ascending order: the scale of soft SUSY breaking $M_{\mathrm{SUSY}}$, the see-saw induced scale $M_{\text {IND }}$, the left-right unification scale $M_{\mathrm{LR}}$, the scale where Pati-Salam symmetry emerges $M_{\mathrm{PS}}$, and the scale of complete gauge-coupling unification $M_{\mathrm{GUT}}$.

$$
\mathrm{E}: \quad M_{\mathrm{SUSY}} \leq M_{\mathrm{IND}} \leq M_{\mathrm{LR}} \leq M_{\mathrm{PS}} \leq M_{\mathrm{GUT}}
$$


Two of those scales can be regarded as free parameters; we may take the lowest ( $M_{\text {IND }}$ ) and highest $\left(M_{\mathrm{GUT}}\right)$ scale for that purpose. The other scales are then fixed by the matching and unification conditions, if they can be satisfied at all.

There are 324 class-E models. In 254 configurations it is possible to implement gaugecoupling unification (GCU), i.e., satisfy all matching and unification conditions. 131 of these configurations allow for a scale $M_{\mathrm{GUT}}>10^{16} \mathrm{GeV} .76$ configurations are able to produce GCU at the Planck scale.

As discussed in the previous section, in class $\mathrm{E}$ the superfield $h$ is not necessarily contained in the spectrum. We found 77 configurations leading to GCU in which the MSSM-like Higgs is $h_{\Phi}$. As mentioned above, most of the configurations have more than one bidoublet at the EWSB scale. Only 29 feature exactly one. There are roughly 50 sets with 1, 2 or 6 bidoublets each and 81 with 4 . Zero or five are generally excluded by our setup. We also find that a larger number of light bidoublets is correlated with lower scales. Especially in the case of six bidoublets, we find that the maximal value for the GUT scale is $M_{\mathrm{GUT}}<10^{16} \mathrm{GeV}$.

Note that in some models of class E, the see-saw scale $M_{\text {IND }}$ is not phenomenologically relevant (cf. table 2), since they do not contain the colored superfield $F$. Thus, we should break down the model space according to the multiplicity of the $F$ multiplet: zero on the one hand (no see-saw scale), one or three on the other hand.

If there is no field $F$, the lowest-lying threshold above the soft SUSY-breaking scale is the scale of left-right-handed unification, $M_{\mathrm{LR}}$. It turns out that, in some models, this scale can be as low as the SUSY scale. At the other end of the spectrum, the complete unification scale $M_{\mathrm{GUT}}$ can vary in the range between $10^{9} \mathrm{GeV}$ and $10^{19} \mathrm{GeV}$.

In the cases of one or three generations of $F$, the see-saw scale can be as low as the soft SUSY-breaking scale, independent of the GUT scale. The upper bound for the see-saw scale is only fixed by the requirement that it is the lowest-lying scale, and is approximately $M_{\text {IND }} \lesssim 10^{16} \mathrm{GeV}$.

As mentioned before, there are configurations fixing all scales. These posses three generations of $h, \Phi, T_{L / R}$ and one generation of $\Sigma$. The multiplicity of $E$ is not fixed. For three generations of $E$ also one or zero generations of $T_{L / R}$ are possible. The LR-scale is in these configurations fixed to $M_{\mathrm{LR}}=7 \times 10^{3} \mathrm{GeV}$ and the PS scale to be $M_{\mathrm{PS}}=10^{9} \mathrm{GeV}$.

Let us now consider in somewhat more detail, the three particular model types described in section 3 .

Type Em: minimal model. In class E, the minimal model is the standard MSSM without Higgs, ${ }^{13}$ supplemented only by the additional fields $\Phi$ and $\Sigma$ above their respective thresholds. Looking at table 2 , we see that this setup does not provide a see-saw scale. Hence, the sub-unification scales depend only on one free parameter, which we take to be $M_{\mathrm{GUT}}$. Figure $3(\mathrm{a})$ shows the variation of the three other scales as a function of $M_{\mathrm{GUT}}$.

For the value of $M_{\mathrm{GUT}} \approx 3 \times 10^{16} \mathrm{GeV}$, all scales approximately coincide. This is the minimal $M_{\text {GUT }}$ value for which GCU is possible in this setup. For this particular parameter point, the GUT symmetry (e.g., $\mathrm{SO}(10)$ ) directly breaks down to the MSSM by virtue of

\footnotetext{
${ }^{13}$ The electroweak Higgs is contained in $\Phi_{L}$.
} 


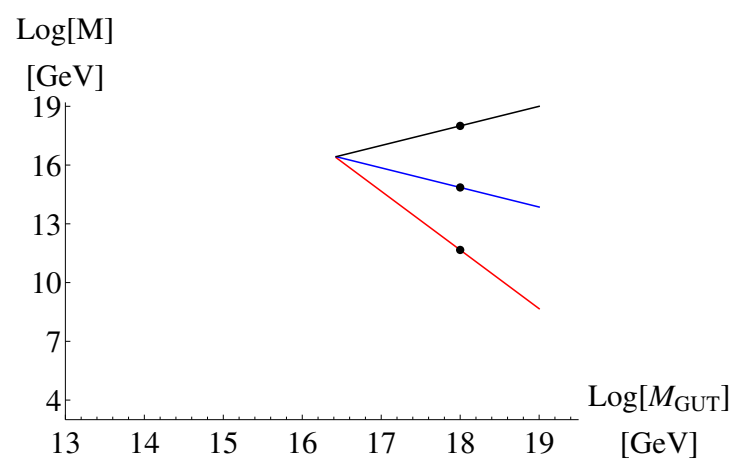

(a) Possible scale variation leading to GCU. The GUT-scale is shown in black, the PS-scale in blue and the LR-scale in red. A IND-scale is not present in this type. The dots indicate the scales for the exemplary plot shown in (b).

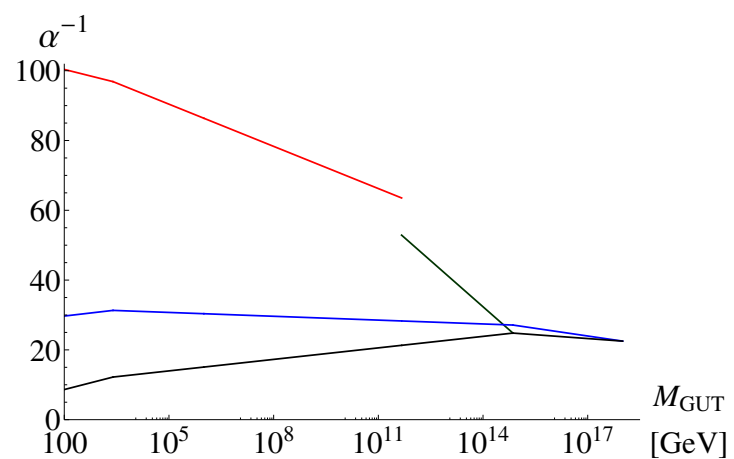

(b) Exemplary running of the gauge couplings for complete unification at $M_{\mathrm{GUT}}=10^{18} \mathrm{GeV}$. The hypercharge coupling is shown in red, the $B-L$ in green, the weak in blue and the strong coupling in black.

Figure 3. Variation of the unification scales and exemplary running of the gauge couplings for the type Em.

$v_{\Sigma}=v_{\Phi}$, so this is actually the standard $\mathrm{SO}(10)$ scenario. If we demand a larger GUT scale, the Pati-Salam scale decreases but never drops below $M_{\mathrm{PS}} \gtrsim 10^{14} \mathrm{GeV}$. The leftright unification scale can vary in the range $10^{11} \mathrm{GeV} \lesssim M_{\mathrm{LR}} \lesssim 10^{16} \mathrm{GeV}$. This is within the favored mass range for right-handed neutrinos. A sample unification plot is shown in figure 3(b).

Type Es: SO(10)-like models. We now turn to a model with complete $\mathrm{SO}(10)$ representations below the GUT scale. With this spectrum, we can vary independently $M_{\text {IND }}$ and $M_{\mathrm{GUT}}$, within a certain range.

It turns out that $M_{\mathrm{GUT}}$, in this type of model, cannot reach the Planck scale. The maximal allowed value for $M_{\mathrm{GUT}}$ depends on $M_{\mathrm{IND}}$ and decreases with increasing $M_{\mathrm{IND}}$. The value of $M_{\text {IND }}$, and thus the mass of the color-triplet fields $F$, can be as low as the soft SUSY-breaking scale.

Another important difference is that the scales approach each other when $M_{\text {GUT }}$ gets larger. In figure 4 , we plot the variation of the sub-unification scales as function of $M_{\mathrm{GUT}}$ for three fixed values of $M_{\text {IND }}$ ( $\operatorname{solid} M_{\text {IND }}=10^{3.4} \mathrm{GeV}$, dashed $M_{\text {IND }}=10^{5.4} \mathrm{GeV}$ and dotted $M_{\text {IND }}=10^{7.4} \mathrm{GeV}$ ). For the lowest value of $M_{\text {IND }}$, it is possible to have GCU without any sub-unification. For larger $M_{\mathrm{IND}}$ we see a gap opening between $M_{\mathrm{LR}}$ and $M_{\mathrm{PS}}$, but it is still possible to achieve $M_{\mathrm{PS}}=M_{\mathrm{GUT}}$.

Type Ee: $\boldsymbol{E}_{\boldsymbol{6}}$-inspired models. In this model, we combine complete $\mathrm{SO}(10)$ multiplets with three generations of the "MSSM-like" Higgs fields $h$ and the color-triplets $F$, so within each generation, matter fields unify with the MSSM Higgs fields and an additional singlet each, to complete $\mathbf{2 7}$ representations of $E_{6}$. In this scenario, GCU is possible over a wide range of mass scales. 


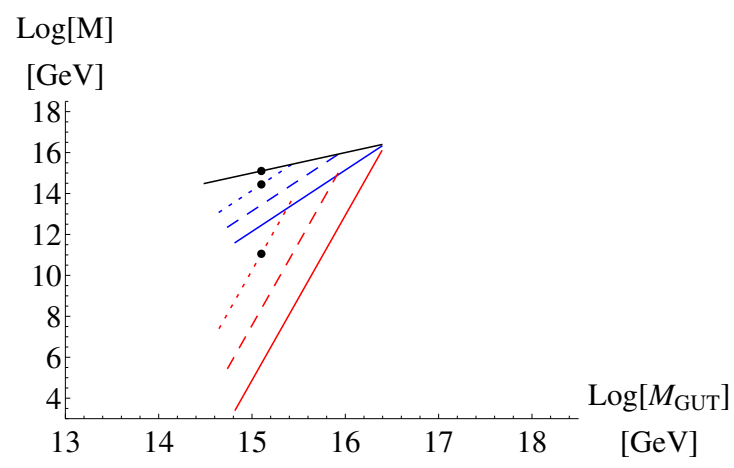

(a) Possible scale variation leading to GCU. The GUT-scale is shown in black, the PS-scale in blue and the LR-scale in red. The variation in the INDscale is shown discrete with $M_{\text {IND }}=10^{3.4} \mathrm{GeV}$ as solid lines, $M_{\text {IND }}=10^{5.4} \mathrm{GeV}$ dashed $M_{\text {IND }}=$ $10^{7.4} \mathrm{GeV}$ dotted. The dots indicate the scales for the exemplary plot shown in (b).

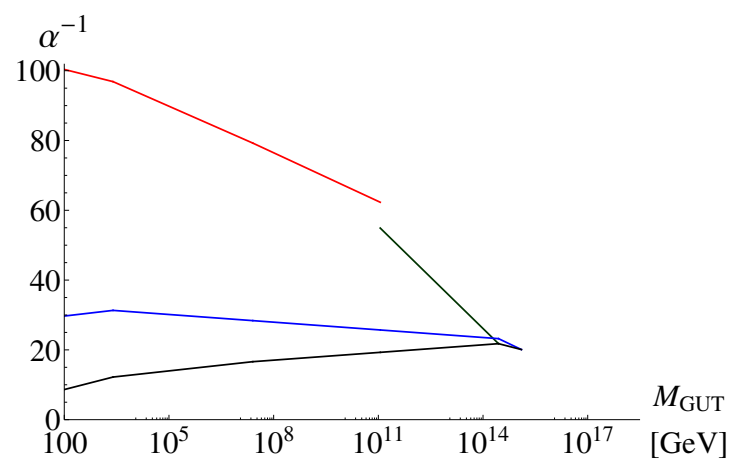

(b) Exemplary running of the gauge couplings for complete unification at $M_{\mathrm{GUT}}=10^{15.1} \mathrm{GeV}$. The hypercharge coupling is shown in red, the $B-L$ in green, the weak in blue and the strong coupling in black.

Figure 4. Variation of the unification scales and exemplary running of the gauge couplings for the type Es.

Like in the model Es discussed above, the separation between the sub-unification scales decreases with increasing scale $M_{\mathrm{GUT}}$. Over the whole range of $M_{\mathrm{IND}}$ it is possible to have the PS and GUT unification coincide, $M_{\mathrm{PS}}=M_{\mathrm{GUT}}$. Complete unification at a single scale is possible for $M_{\mathrm{GUT}} \approx 10^{16.4} \mathrm{GeV}$ if the scale of light triplets is equal to the soft SUSY-breaking scale, $M_{\mathrm{F}}=M_{\mathrm{SUSY}}=2.5 \times 10^{3} \mathrm{GeV}$. This is the well known SU(5) limiting case, since all fields of the low energy spectrum can be grouped to complete SU(5) representations.

Compared to the previous two model types, the gauge coupling at the unification point $\alpha_{\text {GUT }}^{-1}$ is significantly lower and, in some cases, touches the non-perturbative regime. In figure 5 , we show the variation of scales and an exemplary unification plot.

\subsection{Class F}

This model class has a more restricted phenomenology. Nevertheless, this class contain some models that exhibit GCU.

In class-F models, $\mathrm{SU}(2)_{R}$ (and thus LR symmetry) is broken at $v_{T}$, above the scale $v_{\Phi}$ where $\mathrm{SU}(4)_{C}$ reduces to color. We therefore might expect closer relations between leptonflavor and quark-flavor mixing. The relevant scales of this class are, in ascending order: the see-saw induced scale $M_{\mathrm{IND}}$, the quark-lepton unification scale $M_{\mathrm{QL}}$, the Pati-Salam scale $M_{\mathrm{PS}}$, and the unification scale $M_{\mathrm{GUT}}$.

$$
\mathrm{F}: \quad M_{\mathrm{SUSY}} \leq M_{\mathrm{IND}} \leq M_{\mathrm{QL}} \leq M_{\mathrm{PS}} \leq M_{\mathrm{GUT}}
$$

Table 2 indicates that all models in this class do have the additional see-saw scale $M_{\text {IND. }}$. The intermediate scales tend to be higher than in class $\mathrm{E}$ above. 


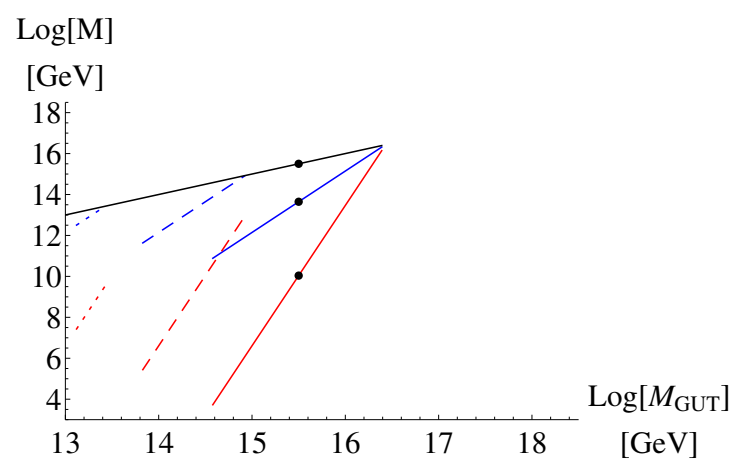

(a) Possible scale variation leading to GCU. The GUT-scale is shown in black, the PS-scale in blue and the LR-scale in red. The variation in the INDscale is shown discrete with $M_{\text {IND }}=10^{3.4} \mathrm{GeV}$ as solid lines, $M_{\text {IND }}=10^{5.4} \mathrm{GeV}$ dashed $M_{\text {IND }}=$ $10^{7.4} \mathrm{GeV}$ dotted. The dots indicate the scales for the exemplary plot shown in (b).

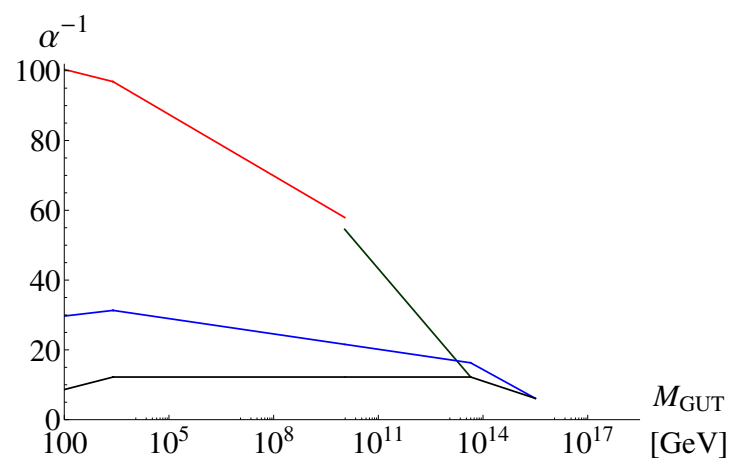

(b) Exemplary running of the gauge couplings for complete unification at $M_{\mathrm{GUT}}=10^{15.1} \mathrm{GeV}$. The hypercharge coupling is shown in red, the $B-L$ in green, the weak in blue and the strong coupling in black.

Figure 5. Variation of the unification scales and exemplary running of the gauge couplings for the type Ee.

Out of the 216 class-F models only 29 configurations are consistent with GCU. In all cases, $M_{\mathrm{GUT}}$ can be as large as the Planck scale.

As discussed in the overview, light degrees of freedom are not possible in this class. As above, we break down the set of configurations according to their content of light fields. There are now 6 categories. In addition to the ones mentioned above (zero, one, or three generations of $F$ ), we have to distinguish cases of one or three generations of $\mathrm{SU}(2)$ triplets $T_{L}$.

The minimal $M_{\text {IND }}$ value is strongly dependent on the number of $\mathrm{SU}(2)$ triplets. In the case of three triplets, it is strictly larger than $10^{16} \mathrm{GeV}$, essentially independent of the number of $F$ fields. Thus, let us look at the configurations with only one $T_{L / R}$ generation.

In these configurations, $M_{\text {IND }}$ is bound to be larger than $M_{\text {IND }} \gtrsim 10^{6} \mathrm{GeV}$. It is realized for three generations of $F$ and rises the less are included.

We conclude that in class $\mathrm{F}$, the extra fields may play a role for flavor physics in an intermediate energy range, but are unlikely to be observable in collider experiments.

Type Fm: minimal model. The minimal model of class F contains the superfields $\Phi$ and $T_{L / R}$ in addition to the MSSM spectrum.

In models of this type, the lowest possible see-saw mass value is $M_{\text {IND }} \approx 10^{12}$ and LR-scale is $M_{\mathrm{LR}} \gtrsim 10^{15}$. Thus these are ruled out, since the mass of the EWSB Higgs is associated to the LR-scale. A next to minimal setup explicitly including one generation of $h$ is not able to produce GCU.

Type Fs/Fe. In type Fs, there is no model consistent with GCU. This is because $\alpha_{3}^{-1}$ grows to fast and overshoots $\alpha_{2}^{-1}$ before the condition (6.4b) for a possible QL-scale can be fulfilled. A model of type Fe consistent with GCU is also not possible. 
Type Ff: flavor-symmetry inspired model. In the absence of the previous types, we take a look at a configuration which might be viewed as $E_{6}$-inspired, but with the additional condition that two of three Higgs bidoublets get heavy (Planck scale) by some unspecified mechanism. In this model, unification is possible over a wide range of mass scales. There is a strong correlation of $M_{\mathrm{IND}}$ and $M_{\mathrm{GUT}}$, so the latter is the only relevant parameter. $M_{\text {IND }}$ can vary between $10^{7} \mathrm{GeV} \lesssim M_{\text {IND }} \lesssim 10^{16} \mathrm{GeV}$. For its largest allowed value all scales are approximately equal, which is the $\mathrm{SO}(10)$ limiting case. Conversely, the lowest possible $M_{\text {IND }}$ value corresponds to GUT unification near the Planck scale. The QL, the PS and the GUT scales are nearly degenerate in any case.

As mentioned above, there is no possible configuration leading to GCU with three generations of the field $h$.

\subsection{Classes A to D}

In these classes, we effectively combine model classes $\mathrm{E}$ and $\mathrm{F}$. There are five different scales, two of which are fixed by requiring GCU. For concreteness, we also fix $M_{\mathrm{GUT}}=$ $10^{18.2} \mathrm{GeV}$, i.e., we assume complete unification at the Planck scale. Still, we can choose two parameters independently, so we will obtain allowed and forbidden regions, but no one-to-one correspondences.

More specifically, we distinguish the cases $v_{T} \leq v_{\Sigma}$ (class B) and $v_{T}>v_{\Sigma}$ (class C), where A and D appear as limits. The ordering of scales in the two scenarios is

$$
\begin{array}{ll}
\mathrm{B}: & M_{\mathrm{SUSY}} \leq M_{\mathrm{IND}} \leq M_{\mathrm{U} 1} \leq M_{\mathrm{LR}} \leq M_{\mathrm{PS}} \leq M_{\mathrm{GUT}} \\
\mathrm{C}: & M_{\mathrm{SUSY}} \leq M_{\mathrm{IND}} \leq M_{\mathrm{U} 1} \leq M_{\mathrm{QL}} \leq M_{\mathrm{PS}} \leq M_{\mathrm{GUT}}
\end{array}
$$

Here, $M_{\mathrm{U} 1}$ indicates the mass scale where the extra U(1) groups break down to hypercharge. This is also the natural scale for a mass term of the right-handed neutrinos. The spectrum below $M_{\mathrm{U} 1}$ still contains the extra particles that are integrated out at the lower see-saw scale $M_{\mathrm{IND}}$, which in turn is located above the soft SUSY-breaking scales. The labels LR and QL refer to left-right and quark-lepton symmetry breaking, respectively.

Of the 144 models in classes B and C, 18 (B) and 57 (C) are consistent with GCU, respectively. We observe again that the number of $T_{L}$ generations has a strong impact on phenomenology. First, we look at the case of three $T_{L}$ generations. In class $\mathrm{C}, M_{\text {IND }}$ depends on the number of generations of the field $F$. It ranges from $M_{\text {IND }} \gtrsim 10^{15} \mathrm{GeV}$ (no $F$ ) down to to $M_{\text {IND }} \gtrsim 10^{6} \mathrm{GeV}$ (three $F$ generations). The situation in class B is even worse. Here GCU is not possible with less then three generations of the field $F$.

If there is only one generation of $T$, the $M_{\text {IND }}$ value can approach the SUSY scale, independent on the number of $F$.

Type Bm/Cm: minimal model. In the minimal model, there is a single generation of each of $\Phi, T_{L / R}$ and $\Sigma$. We can achieve GCU for both Bm and Cm. The see-saw scale is stuck at rather high values, $M_{\mathrm{IND}} \gtrsim 10^{13} \mathrm{GeV}$ and $M_{\mathrm{IND}} \gtrsim 10^{10} \mathrm{GeV}$ for type Bm and $\mathrm{Cm}$, respectively. Since we again do not explicitly add a EWSB Higgs, such models are ruled out because of the nonexistence of light $\mathrm{SU}(2)$ doublets. If we include one generation of $h$ in addition, GCU is no longer possible. 


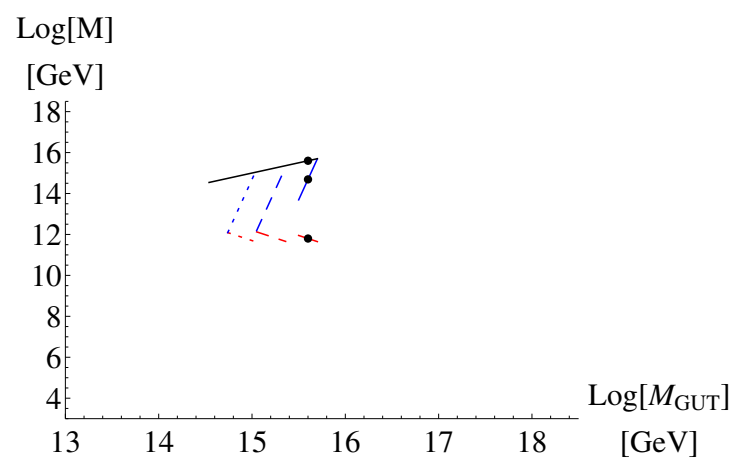

(a) Possible scale variation leading to GCU. The PS-scale is shown in black, the LR-scale in blue and the MSSM-scale in red. The variation in the IND-scale is shown discrete with $M_{\text {IND }}=10^{4} \mathrm{GeV}$ as solid lines, $M_{\mathrm{IND}}=10^{7} \mathrm{GeV}$ dashed $M_{\mathrm{IND}}=$ $10^{10} \mathrm{GeV}$ dotted. The dots indicate the scales for the exemplary plot shown in (b).

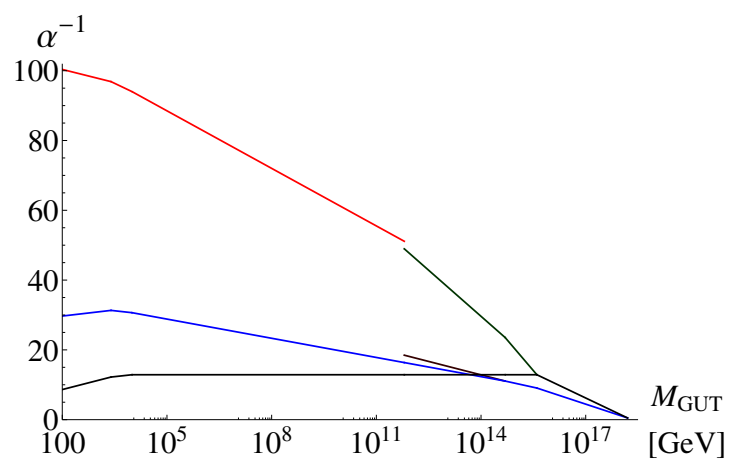

(b) Exemplary running of the gauge couplings for complete unification at $M_{\mathrm{GUT}}=10^{18.2} \mathrm{GeV}$. The hypercharge coupling is shown in red, the $\mathrm{U}(1)_{R}$ in brown, the $B-L$ in green, the weak in blue and the strong coupling in black.

Figure 6. Variation of the unification scales and exemplary running of the gauge couplings for the type B199.

Types Bs/Cs and Be/Ce. These setups do not allow GCU.

Class-B/C models with $M_{\text {IND }}<10 \mathrm{TeV}$. We may ask for models where the see-saw scale is sufficiently low (say, $M_{\text {IND }}<10 \mathrm{TeV}$ ) that the new particles can have an impact on collider phenomenology. We find 8 (34) models where this is possible within class B (C), respectively. One configuration with normal hierarchy is model B199, ${ }^{14}$ where we have three copies of $h, F$ and $\Sigma$ and no $E$. In the inverted case there is a similar model C211, which has the same spectrum, but three copies of $\Phi$. The plots are shown in figure 6,7 .

\section{Pati-Salam models without supersymmetry}

We now turn to scenarios without supersymmetry. Obviously, there is much greater freedom for model building, if we ignore the naturalness problems that inevitably appears when there are scalar fields in the spectrum. To limit this freedom in a meaningful way, we consider the same classes of models as in the supersymmetric case, but omit the fermionic superpartners of the additional multiplets. Analogously, we omit the scalar superpartners of matter fields and the fermionic superpartners of gauge fields. This (ad-hoc) restriction allows us to compare supersymmetric and non-supersymmetric models on the same basis. The meaning of scales and symmetry breaking patterns are unchanged.

Since we found that in the non-SUSY case the resulting mass thresholds tend to be lower, we fixed the GUT scale for the classes B and C to $M_{\mathrm{GUT}} \equiv 10^{16} \mathrm{GeV}$. For the value of $M_{\mathrm{GUT}}=10^{18.2} \mathrm{GeV}$ which we used in the SUSY case, we would have only one

\footnotetext{
${ }^{14}$ For the meaning of numerical model indices, see appendix C.
} 


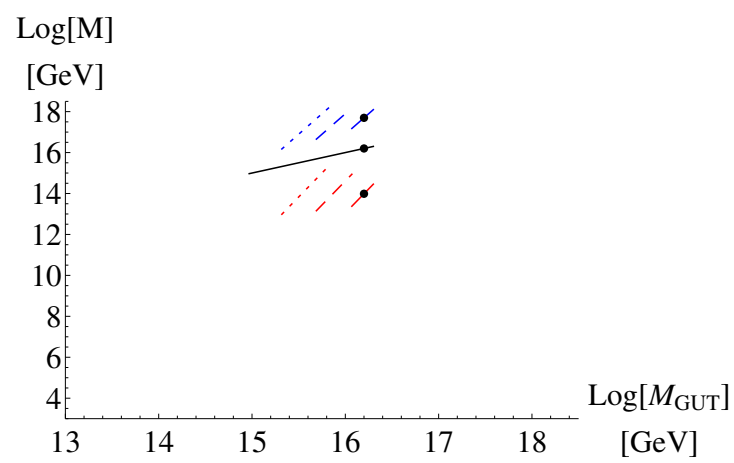

(a) Possible scale variation leading to GCU. The QL-scale is shown in black, the PS-scale in blue and the MSSM-scale in red. The variation in the IND-scale is shown discrete with $M_{\text {IND }}=10^{4} \mathrm{GeV}$ as solid lines, $M_{\mathrm{IND}}=10^{7} \mathrm{GeV}$ dashed $M_{\mathrm{IND}}=$ $10^{10} \mathrm{GeV}$ dotted. The dots indicate the scales for the exemplary plot shown in (b).

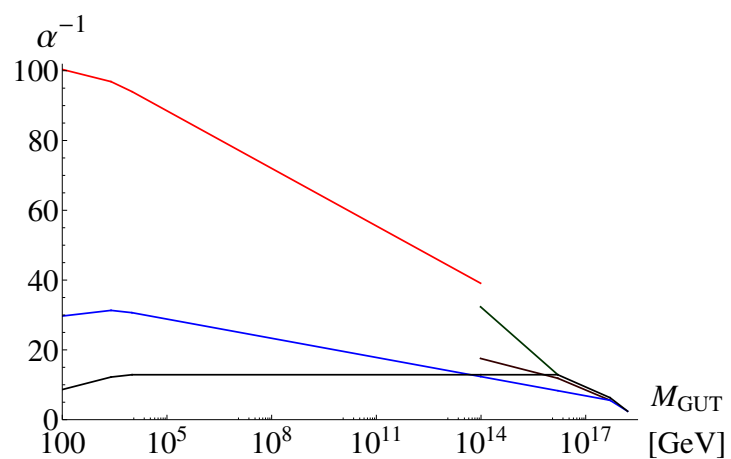

(b) Exemplary running of the gauge couplings for complete unification at $M_{\mathrm{GUT}}=10^{18.2} \mathrm{GeV}$. The hypercharge coupling is shown in red, the $\mathrm{U}(1)_{R}$ in brown, the $B-L$ in green, the weak in blue and the strong coupling in black.

Figure 7. Variation of the unification scales and exemplary running of the gauge couplings for the type C211.

configuration (class C) for both classes that satisfy GCU. Lowering this scale even more, the model space would be less constrained, but this is disfavored by the proton decay limits.

We considered the same set of 828 models, with the SUSY partners removed, as in the previous section. Again, more than half of the models allow for GCU, given the modification mentioned in the previous paragraph. Still it turns out that without SUSY, classes A-D are disfavored but not excluded. In contrast to the supersymmetric case, a considerable set of the successful models fall in class F. Again, the most belong to class E. In any case, lower GUT scales tend to be favored.

As in the SUSY models discussed above, we observe a LR symmetry breaking scale roughly around $10^{13} \mathrm{GeV}$ in a large fraction of the successful models. Nevertheless, there are models where this scale can be much lower, down to below $100 \mathrm{TeV}$ in some cases.

In class $\mathrm{E}$, there are again many models with the possibility for light color triplets $F$ in the $\mathrm{TeV}$ regime. In the non-SUSY setup, these are scalar particles and obviously cannot mix with quarks. We have to assume that there are couplings of either leptoquark or diquark type that render these particles unstable, originating from the PS-breaking sector. Furthermore, in models of this kind there is a high probability that the MSSM Higgs is not part of $h$ but of the $h_{\Phi}$ multiplet (see section 5.3). Similar to the SUSY case the number of light bidoublets is constrained to be one in the classes A to D. For class $\mathrm{E}$ we found also a similar result as in the SUSY case. Again, most configurations prefer four generations. Six are somehow disfavored, and one to three are equally likely. In contrast to the previous considerations, there are now plenty of configurations of class $\mathrm{F}$ with three generations of bidoublets, but still one is in favor here. Again we see, that the multiplicity of these bidoublets lowers the maximal unification scale. 


\begin{tabular}{|l|c|c|c|c|c|}
\hline & class B & class C & class E & class F & $\sum$ \\
\hline scanned & 144 & 144 & 324 & 216 & 828 \\
GCU & 10 & 30 & 230 & 201 & 471 \\
$M_{\mathrm{GUT}}>10^{16} \mathrm{GeV}$ & 10 & 30 & 111 & 16 & 167 \\
$M_{\mathrm{IND}}<10 \mathrm{TeV}$ and $M_{\mathrm{GUT}}>10^{16} \mathrm{GeV}$ & 1 & 8 & 110 & 16 & 135 \\
$M_{\mathrm{LR}}<100 \mathrm{TeV}$ & 0 & 0 & 136 & 0 & 136 \\
$10^{12} \mathrm{GeV}<M_{N_{R}}<10^{14} \mathrm{GeV}$ & 9 & 30 & 211 & 126 & 376 \\
$M_{\mathrm{IND}} \in[0.1,10] \frac{v_{\Phi}^{2}}{v_{\Sigma}+v_{T}}$ & 12 & 36 & 201 & 93 & 342 \\
\hline
\end{tabular}

Table 4. Number of configurations full filling certain conditions in the non-SUSY case.

As in the SUSY case, we find that three generations of light $\mathrm{SU}(2)$ triplets $T$ are excluded. In particular, in class $\mathrm{C}$ the lower bound for those is $M_{T_{L}} \gtrsim 10^{8} \mathrm{GeV}$. In class $\mathrm{C}$, the bound becomes $10^{11} \mathrm{GeV}$ and in class $\mathrm{B}$ there is no GCU at all.

In the non-supersymetric case we again find in all classes a set of configurations fixing all mass scales "exactly". This usually corresponds to degenerate mass scales. A common scale for such classes is $M_{\mathrm{GUT}} \approx 10^{14} \mathrm{GeV}$.

Again we find that fixing the induced scale $M_{\text {IND }}$ does not change the results very much. Thus we see our assumptions justified to include this scale in our scans.

A general feature of non-SUSY spectra is the fact that the high-energy effective values of the gauge couplings are larger than in the SUSY case. This is due to the lower number of fields that contribute to the gauge-coupling running.

In the following, we do not repeat the detailed discussion of SUSY models but pick a few selected models and model types with particular features. More generic statistics can be read off table 4 .

\subsection{Class $\mathbf{E}$}

For class E it is possible to implement GCU in 230 configurations, of which 23 provide a complete unification near the Planck scale. Similar to the supersymmetric case, we find 88 configurations where the EWSB Higgs is provided by $\Phi_{L}$. On the other hand, an interesting possibility is the existence of three Higgs generations (type Ee). Although, in the non-SUSY case, there is no direct relation to $E_{6}$ unification, we may take a look at such models. In class E, we find that GCU is possible, and the GUT-scale can vary between $10^{14} \mathrm{GeV} \lesssim M_{\mathrm{GUT}} \lesssim 10^{17} \mathrm{GeV}$. One possible configuration exhibits three generations of $F$, one of $\Sigma$ and $\Phi$ each, and no fields $E$ or $T$. For this special configuration the possible variation of the scales and the unification plot are shown in figure 8 . Here we find that the variation of the LR-scale strongly depends on the GUT-scale. The PS scale varies only weakly and is always close to the GUT scale.

\subsection{Class F}

In class F, there are plenty of configurations leading to successful GCU. In general, we find that there is not much scope for scale variation. The GUT scale can be as large as 


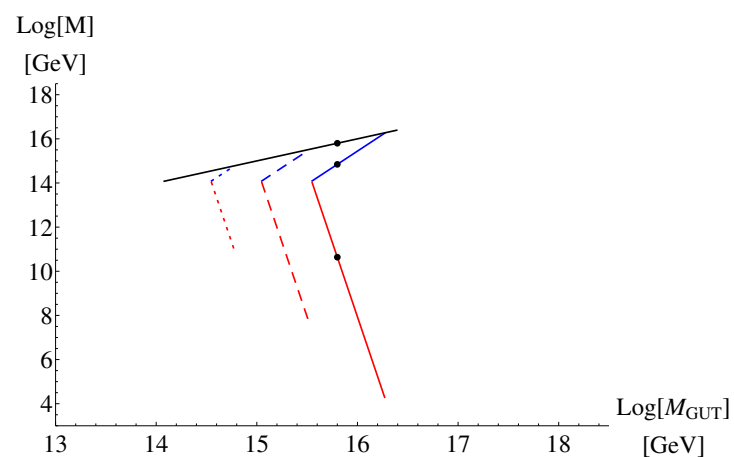

(a) Possible scale variation leading to GCU. The GUT-scale is shown in black, the PS-scale in blue and the LR-scale in red. The variation in the IND-scale is shown discrete with $M_{\text {IND }}=10^{4} \mathrm{GeV}$ as solid lines, $M_{\mathrm{IND}}=10^{7} \mathrm{GeV}$ dashed $M_{\mathrm{IND}}=$ $10^{10} \mathrm{GeV}$ dotted. The dots indicate the scales for the exemplary plot shown in (b).

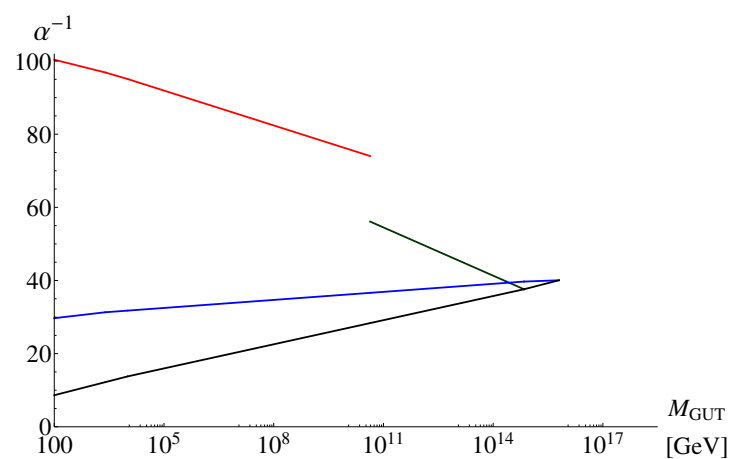

(b) Exemplary running of the gauge couplings for complete unification at $M_{\mathrm{GUT}}=10^{15.8} \mathrm{GeV}$. The hypercharge coupling is shown in red, the $B-L$ in green, the weak in blue and the strong coupling in black.

Figure 8. Variation of the unification scales and exemplary running of the gauge couplings for the non-SUSY type Ee.

$M_{\mathrm{GUT}} \approx 10^{17} \mathrm{GeV}$. The scales are close to each other, since the lightest scale is fixed to be larger than $M_{\text {IND }} \gtrsim 10^{13} \mathrm{GeV}$. In this class there are also models with GCU where all scales are essentially fixed, and not far from the GUT scale. Those lead to $M_{\mathrm{GUT}} \approx 2 \times 10^{14} \mathrm{GeV}$, which is rather low. The LR-scale emerges between $10^{13} \mathrm{GeV} \leq M_{\mathrm{LR}} \leq 2 \times 10^{15} \mathrm{GeV}$.

One exemplary configuration leading to GCU above $10^{16} \mathrm{GeV}$ is model F213. Here, we have three generations of $F$ and one of $T$. These scalar particles can be rather light, potentially as low as the SUSY scale. In addition, this model contains three generations of the fields $\Phi$ and $\Sigma$, and one generation of $E$. We show the possible scale variation and a sample unification plot for this model in figure 9 .

\subsection{Class A to D}

In class C, the QL-scale emerges typically close the PS scale. Likewise, the PS scale can become as large as the GUT-scale, such that the energy range with pure PS symmetry may vanish.

Looking at the possibility of three Higgs $(h)$ generations, we do not find any configurations in class $\mathrm{B}$, and a few in class $\mathrm{C}$. On the other hand, these model classes favor three as the number of $\Phi$ generations.

In figure 10 and 11, we display the scale relations and gauge-coupling unification for two distinct models B53 and C45. ${ }^{15}$ The former model contains three generations of $F$ which can be light. In addition it contains three generations of $E$ and all other fields ones. We found quite some range for scale variation. For the largest value of the PS scale

\footnotetext{
${ }^{15}$ For the meaning of numerical model indices, see appendix C.
} 


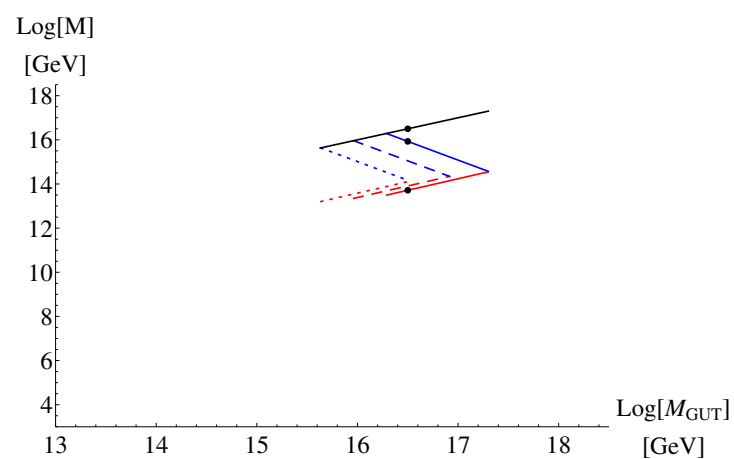

(a) Possible scale variation leading to GCU. The GUT-scale is shown in black, the PS-scale in blue and the QL-scale in red. The variation in the INDscale is shown discrete with $M_{\text {IND }}=10^{4} \mathrm{GeV}$ as solid lines, $M_{\mathrm{IND}}=10^{8.5} \mathrm{GeV}$ dashed $M_{\mathrm{IND}}=$ $10^{13} \mathrm{GeV}$ dotted. The dots indicate the scales for the exemplary plot shown in (b).

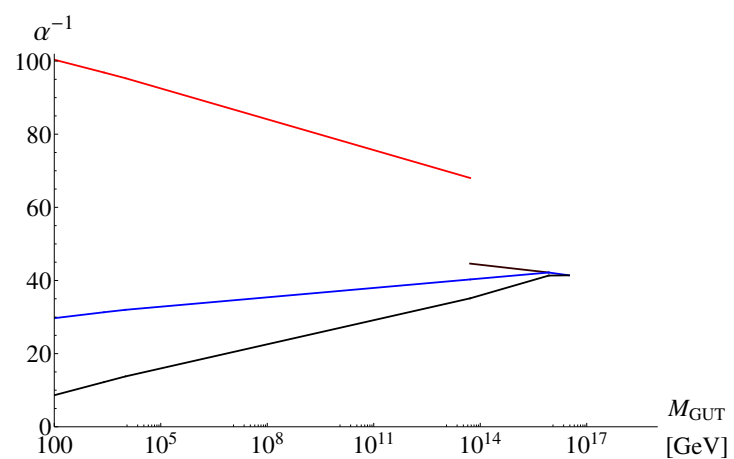

(b) Exemplary running of the gauge couplings for complete unification at $M_{\mathrm{GUT}}=10^{16.5} \mathrm{GeV}$. The hypercharge coupling is shown in red, the $\mathrm{U}(1)_{R}$ in brown, the weak in blue and the strong coupling in black.

Figure 9. Variation of the unification scales and exemplary running of the gauge couplings for the type non-SUSY F213.

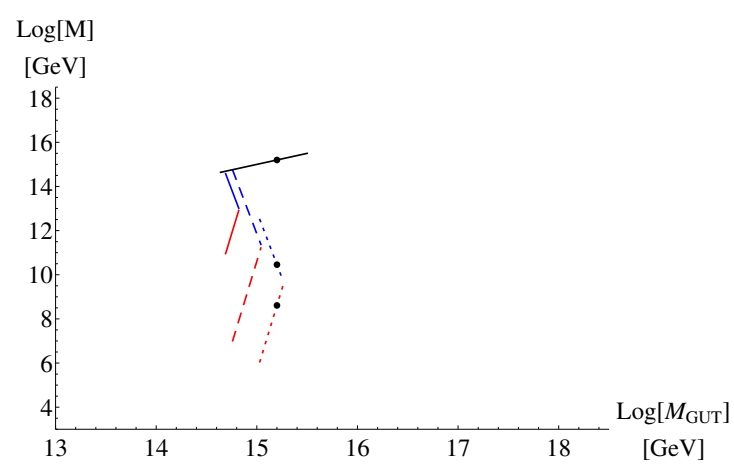

(a) Possible scale variation leading to GCU. The PS-scale is shown in black, the LR-scale in blue and the MSSM-scale in red. The variation in the IND-scale is shown discrete with $M_{\text {IND }}=10^{4} \mathrm{GeV}$ as solid lines, $M_{\mathrm{IND}}=10^{5} \mathrm{GeV}$ dashed $M_{\mathrm{IND}}=$ $10^{6} \mathrm{GeV}$ dotted. The dots indicate the scales for the exemplary plot shown in (b).

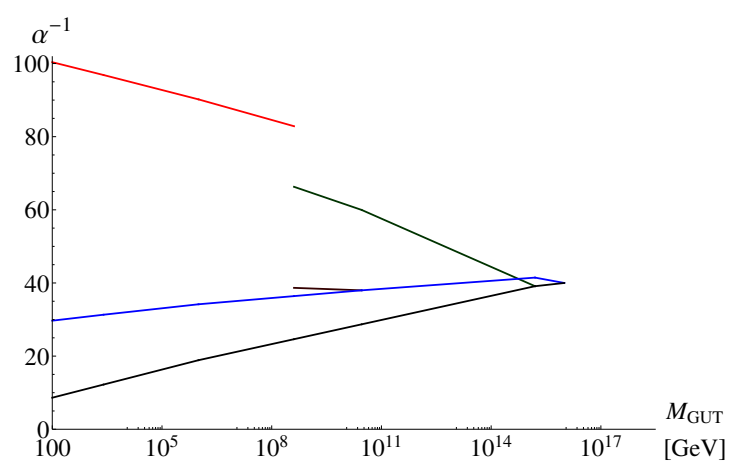

(b) Exemplary running of the gauge couplings for complete unification at $M_{\mathrm{GUT}}=10^{16} \mathrm{GeV}$. The hypercharge coupling is shown in red, the $\mathrm{U}(1)_{R}$ in brown, the $B-L$ in green, the weak in blue and the strong coupling in black.

Figure 10. Variation of the unification scales and exemplary running of the gauge couplings for the non-SUSY type B25.

$\left(M_{\mathrm{PS}} \approx 10^{15}\right)$ we find a degeneracy of all lower scales. The induced scale can be as light as some $\mathrm{TeV}$.

Model C45 also features new particles at the TeV scale. Here we include three generations of $\Phi$ and $\Sigma$ and all other fields ones. We again see quite some space to vary the scales. 


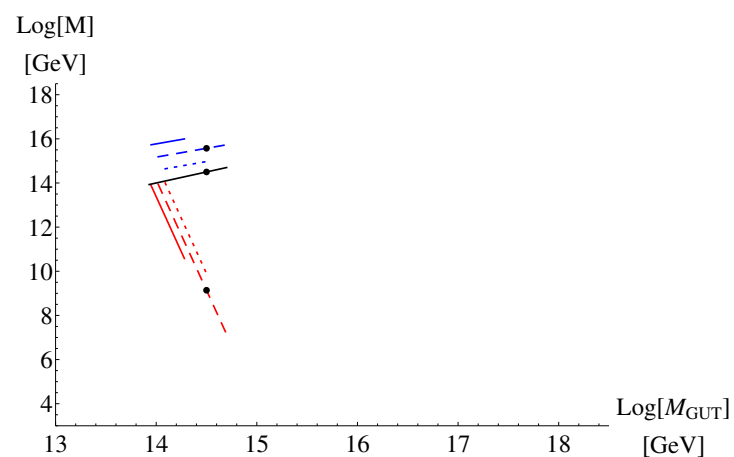

(a) Possible scale variation leading to GCU. The QL-scale is shown in black, the PS-scale in blue and the MSSM-scale in red. The variation in the IND-scale is shown discrete with $M_{\text {IND }}=10^{4} \mathrm{GeV}$ as solid lines, $M_{\mathrm{IND}}=10^{7} \mathrm{GeV}$ dashed $M_{\mathrm{IND}}=$ $10^{10} \mathrm{GeV}$ dotted. The dots indicate the scales for the exemplary plot shown in (b).

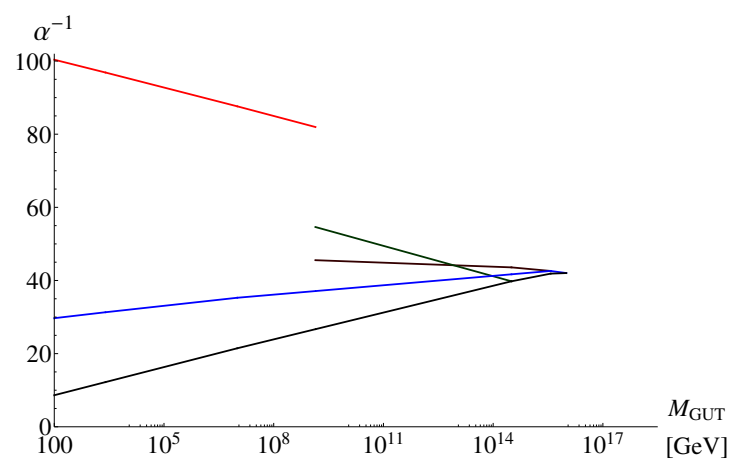

(b) Exemplary running of the gauge couplings for complete unification at $M_{\mathrm{GUT}}=10^{16} \mathrm{GeV}$. The hypercharge coupling is shown in red, the $\mathrm{U}(1)_{R}$ in brown, the $B-L$ in green, the weak in blue and the strong coupling in black.

Figure 11. Variation of the unification scales and exemplary running of the gauge couplings for the non-SUSY type C27.

\section{$9 \quad$ Summary of models}

We have presented a survey of models with gauge-coupling unification along a path that contains several intermediate scales, corresponding to left-right symmetry, quark-lepton unification, Pati-Salam symmetry, and SO(10) or larger GUT symmetry. We studied both supersymmetric and non-supersymmetric models, where the latter are derived from the former by omitting all superpartners.

We have restricted the allowed new chiral superfields (or scalar fields, in the nonSUSY case) below the GUT scale to a small well-defined set of multiplets, all of which fit in small representations of $\mathrm{SO}(10)$ or $E_{6}$. A large subset of the resulting models is consistent with gauge-coupling unification, proceeding in several steps. In supersymmetric models, there is slightly more freedom in varying the scales than in non-supersymmetric models.

The assumptions and calculations do not constrain the model space in such a way that we can get precise numerical predictions, but we can deduce characteristic patterns in the scale hierarchies that correlate with specific choices for the spectrum.

In a wide range of models, GUT unification can be pushed up to the Planck scale. This fact, together with the properties of Pati-Salam symmetry, significantly reduces the strain that the non-observation of proton decay can put on GUT model building.

Additional thresholds in the intermediate range between observable energies and the GUT scale are likely. Being associated with left-right or quark-lepton symmetry breaking, they decouple flavor physics issues from the requirements of complete unification. We have not considered flavor physics in any more detail, but expect that it can generically be accommodated if non-renormalizable terms are properly included. Depending on the 
particular model and on the chosen set of flavor-dependent interactions, we expect specific hierarchies, relations and predictions for the flavor sector.

As an extra feature, the (super)fields $\Sigma$ and $T$ (adjoint of $\mathrm{SU}(4)_{C}$ and $\left.\mathrm{SU}(2)_{R}\right)$ and $\Phi$ (fundamental of $\left.\mathrm{SU}(2)_{L} \times \mathrm{SU}(2)_{R}\right)$ can cooperate to generate small mass terms for certain particles, including SM-like Higgs doublets and new exotic (s)quarks, such that they can be accessible at colliders. On the other hand, $\mathrm{SU}(2)_{L / R}$ triplets as possible Pati-Salam breaking Higgs fields tend to raise the LR symmetry scale above the scale of quark-lepton unification and thus may enforce a direct relation between quark and lepton flavor physics.

Another generic property of the models under consideration is the scale of left-right symmetry breaking, naturally associated with neutrino mass generation, in an intermediate mass range. A neutrino mass scale significantly below the GUT scale is favored by numerical estimates of see-saw mechanisms that can explain the small observable neutrino masses.

We also encounter models where gauge-coupling unification at high energies implies a multi-Higgs doublet model at low scale, possibly with flavor quantum numbers. Furthermore, the observable Higgs doublet need not be a member of a $(1,2,2)$ representation, as often assumed, but can also originate from a $(4,2,1)+$ c.c. representation, i.e., behave as a scalar lepton. The effective $\mu$ term which sets the scale for low-energy Higgs physics, can have a see-saw like form and thus be naturally suppressed with respect to the higher symmetry-breaking scales.

\section{Conclusions}

In summary, we have studied a range of comparatively simple models that fit into the framework of GUT theories with intermediate thresholds. We have taken a phenomenological viewpoint and specified the models in form of a chain of effective theories, as far as we expect that a description in terms of weakly interacting four-dimensional gauge theory can make sense.

It is remarkable that the most interesting phenomenology, which we may identify as Planck-scale GUT unification, intermediate PS and LR scales, and new particles at TeV energies, can be simultaneously realized in a number of distinct models (cf. appendix D). This is not a generic feature. However, if not all of these conditions are to be satisfied simultaneously, or allowing further multiplets or hierarchy patterns in couplings, the set of interesting models becomes sizable.

One may consider fully specified GUT models that predict the appearance (or absence) of a PS symmetry and the associated spectrum. However, the current lack of reliable knowledge about strong and gravitational interactions which are expected at the highest energies, denies attempts to ultimately favor or exclude alternative spectra and symmetrybreaking chains.

In such a situation, it appears worthwhile to rather concentrate on the implications of a sequence of new thresholds at intermediate energies, presumably in the context of PS symmetry. Our survey demonstrates that intermediate symmetry-breaking scales associated with flavor mixing and mass generation can emerge in various energy regions, even if rather specific and simple assumptions about gauge multiplets are imposed. The findings suggest 
that one should study the hierarchies within flavor observables in relation to hierarchies in gauge-symmetry breaking, discuss both renormalizable and non-renormalizable operators, and to combine gauge and flavor symmetries in a common framework which need not be tied to ultimate GUT unification. This program, which has so far been pursued only for a subset of the possible scenarios, deserves further efforts.

In summary, Pati-Salam models can easily accommodate unification in a multi-scale framework. They provide a rich phenomenology and a promising background for new approaches to the lepton and quark flavor problem.

\section{A Beta-function coefficients}

As stated in the paper (cf. section 6) the running of the gauge couplings can be described by

$$
\frac{1}{\alpha_{i}\left(\mu_{2}\right)}=\frac{1}{\alpha_{i}\left(\mu_{1}\right)}-\frac{b_{i}}{2 \pi} \ln \left(\frac{\mu_{2}}{\mu_{1}}\right) \text {. }
$$

The coefficient $b_{i}$ of the running coupling can be calculated by means of the representation of the fields contributing at the given mass scale alone [48]. For each set of gauge groups $\mathrm{SU}(N)$ with $N \geq 2$ the contribution of an field with representation $\left(\boldsymbol{I}_{1}, \ldots, \boldsymbol{I}_{n}\right)$ to the running coefficient $\tilde{b}_{i}$ is given as

$$
\tilde{b}_{i}^{\Re}=T\left(\boldsymbol{I}_{i}\right) \prod_{k \neq i} d\left(\boldsymbol{I}_{k}\right),
$$

where $d\left(\boldsymbol{I}_{i}\right)$ is the dimension and the normalization of the representation $T\left(\boldsymbol{I}_{i}\right)$. These can be calculated using the representing matrices $R^{a}$

$$
\operatorname{tr} R^{a} R^{b}=T(\Re) \delta^{a b},
$$

with $T(\boldsymbol{N})=\frac{1}{2}$.

For a $\mathrm{U}(1)$ the contribution is up to an consistent rescaling:

$$
\tilde{b}_{\mathrm{U}(1)}^{\Re}=Y^{2} \prod_{k} d\left(\boldsymbol{I}_{k}\right) .
$$

The complete running coefficient depends now on whether we work in a supersymmetric theory or not. For the non-supersymetric case one has to divide the fields in scalar and fermionic contributions:

$$
b_{i}^{\mathrm{SM}}=\frac{2}{3} \sum_{\Re_{\text {ferm }}} \tilde{b}_{i}^{\Re}+\frac{1}{3} \sum_{\Re_{\text {scalar }}} \tilde{b}_{i}^{\Re}-\frac{11}{3} C_{2}\left(G_{i}\right),
$$

where $C_{2}\left(\boldsymbol{G}_{i}\right)=\operatorname{dim}\left(G_{i}\right)$ is the quadratic Casimir operator.

Since in the supersymmetric case there is a superpartner for each scalar/fermionic field, there is no need to divide the fields in such a way. Thus, the relation simplifies to

$$
b_{i}^{\mathrm{SUSY}}=\sum_{\mathfrak{R}} \tilde{b}_{i}^{\Re}-3 C_{2}\left(G_{i}\right) .
$$

Table 5 displays the contribution of each field and its complete decomposition w.r.t. the subgroups of PS symmetry. 


\begin{tabular}{|c|c|c|c|c|c|c|c|c|}
\hline Field & PS & LR & $\mathrm{SM}$ & $\tilde{b}_{Y}$ & $\tilde{b}_{B-L}$ & $\tilde{b}_{2}$ & $\tilde{b}_{3}$ & $\tilde{b}_{4}$ \\
\hline \multirow[t]{2}{*}{$h$} & $(1,2,2)$ & $(1,2,2)_{0}$ & $(1,2)_{\frac{1}{2}}$ & $\frac{1}{2}$ & 0 & $\frac{1}{2}$ & 0 & 0 \\
\hline & & & $(1,2)_{-\frac{1}{2}}$ & $\frac{1}{2}$ & & $\frac{1}{2}$ & 0 & \\
\hline \multirow[t]{2}{*}{$F$} & $(6,1,1)$ & $(3,1,1)_{\frac{2}{3}}$ & $(3,1)_{\frac{1}{3}}$ & $\frac{1}{3}$ & $\frac{4}{3}$ & 0 & $\frac{1}{2}$ & 1 \\
\hline & & $(\overline{3}, 1,1)_{-\frac{2}{3}}$ & $(\overline{3}, 1)_{-\frac{1}{3}}$ & $\frac{1}{3}$ & $\frac{4}{3}$ & 0 & $\frac{1}{2}$ & \\
\hline \multirow[t]{4}{*}{$\Phi_{R}$} & $(\overline{4}, 1,2)$ & $(\overline{3}, 1,2)_{\frac{1}{3}}$ & $(\overline{3}, 1)_{\frac{2}{3}}$ & $\frac{4}{3}$ & $\frac{2}{3}$ & 0 & $\frac{1}{2}$ & 1 \\
\hline & & & $(\overline{3}, 1)_{-\frac{1}{3}}$ & $\frac{1}{3}$ & & 0 & $\frac{1}{2}$ & \\
\hline & & $(1,1,2)_{-1}$ & $(1,1)_{0}$ & 0 & 2 & 0 & 0 & \\
\hline & & & $(1,1)_{-1}$ & 1 & & 0 & 0 & \\
\hline \multirow{4}{*}{$\bar{\Phi}_{R}$} & $(4,1,2)$ & $(3,1,2)_{-\frac{1}{3}}$ & $(3,1)_{\frac{1}{3}}$ & $\frac{1}{3}$ & $\frac{2}{3}$ & 0 & $\frac{1}{2}$ & 1 \\
\hline & & & $(3,1)_{-\frac{2}{3}}$ & $\frac{4}{3}$ & & 0 & $\frac{1}{2}$ & \\
\hline & & $(1,1,2)_{1}$ & $(1,1)_{1}$ & 1 & 2 & 0 & 0 & \\
\hline & & & $(1,1)_{0}$ & 0 & & 0 & 0 & \\
\hline \multirow[t]{2}{*}{$\Phi_{L}$} & $(4,2,1)$ & $(3,2,1)_{-\frac{1}{3}}$ & $(3,2)_{-\frac{1}{6}}$ & $\frac{1}{6}$ & $\frac{2}{3}$ & $\frac{3}{2}$ & 1 & 1 \\
\hline & & $(1,2,1)_{1}$ & $(1,2)_{\frac{1}{2}}$ & $\frac{1}{2}$ & 2 & $\frac{1}{2}$ & 0 & \\
\hline \multirow[t]{2}{*}{$\bar{\Phi}_{L}$} & $(\overline{4}, 2,1)$ & $(\overline{3}, 2,1)_{\frac{1}{3}}$ & $(\overline{3}, 2)_{\frac{1}{6}}$ & $\frac{1}{6}$ & $\frac{2}{3}$ & $\frac{3}{2}$ & 1 & 1 \\
\hline & & $(1,2,1)_{-1}$ & $(1,2)_{-\frac{1}{2}}$ & $\frac{1}{2}$ & 2 & $\frac{1}{2}$ & 0 & \\
\hline \multirow[t]{4}{*}{$\Sigma$} & $(15,1,1)$ & $(8,1,1)_{0}$ & $(8,1)_{0}$ & 0 & 0 & 0 & 3 & 4 \\
\hline & & $(3,1,1)_{-\frac{4}{3}}$ & $(3,1)_{-\frac{2}{3}}$ & $\frac{4}{3}$ & $\frac{16}{3}$ & 0 & $\frac{1}{2}$ & \\
\hline & & $(\overline{3}, 1,1)_{\frac{4}{3}}$ & $(\overline{3}, 1)_{\frac{2}{3}}$ & $\frac{4}{3}$ & $\frac{16}{3}$ & 0 & $\frac{1}{2}$ & \\
\hline & & $(1,1,1)_{0}$ & $(1,1)_{0}$ & 0 & 0 & 0 & 0 & \\
\hline \multirow[t]{4}{*}{$E$} & $(6,2,2)$ & $(3,2,2)_{\frac{2}{3}}$ & $(3,2)_{\frac{5}{6}}$ & $\frac{25}{6}$ & $\frac{16}{3}$ & $\frac{3}{2}$ & 1 & 4 \\
\hline & & & $(3,2)_{\frac{1}{6}}$ & $\frac{1}{6}$ & & $\frac{3}{2}$ & 1 & \\
\hline & & $(\overline{3}, 2,2)_{-\frac{2}{3}}$ & $(\overline{3}, 2)_{-\frac{1}{6}}$ & $\frac{1}{6}$ & $\frac{16}{3}$ & $\frac{3}{2}$ & 1 & \\
\hline & & & $(\overline{3}, 2)_{-\frac{5}{6}}$ & $\frac{25}{6}$ & & $\frac{3}{2}$ & 1 & \\
\hline \multirow[t]{3}{*}{$T^{R}$} & $(1,1,3)$ & $(1,1,3)_{0}$ & $(1,1)_{1}$ & 0 & 0 & 0 & 0 & 0 \\
\hline & & & $(1,1)_{0}$ & 0 & 0 & 0 & 0 & 0 \\
\hline & & & $(1,1)_{-1}$ & 0 & 0 & 0 & 0 & 0 \\
\hline$T^{L}$ & $(1,3,1)$ & $(1,3,1)_{0}$ & $(1,3)_{0}$ & 0 & 0 & 2 & 0 & 0 \\
\hline
\end{tabular}

Table 5. Full field content and breaking as well as all contributions to the beta function.

\section{B Vacuum expectation values and mass scales}

In the first part of this paper we calculate the superpotential and the masses of all superfields. Therefore we use the vevs as natural scales. Since in this part the breaking associated to the vev is not the most important thing but the fields they are related to, we name them after those.

In the second part, we are primarily interested in the scales present in the running of the gauge couplings. Hence, we switch our notation to the mass scales. These are labeled 


\begin{tabular}{|ccccc|}
\hline vev & class B & class C & class E & class F \\
\hline$v_{\Sigma}$ & $M_{\mathrm{PS}}$ & $M_{\mathrm{QL}}$ & $M_{\mathrm{PS}}$ & - \\
$v_{T}$ & $M_{\mathrm{LR}}$ & $M_{\mathrm{PS}}$ & - & $M_{\mathrm{PS}}$ \\
$v_{\Phi}$ & $M_{\mathrm{MSSM}}$ & $M_{\mathrm{MSSM}}$ & $M_{\mathrm{LR}}$ & $M_{\mathrm{LR}}$ \\
$\frac{v_{\Phi}^{2}}{v_{\Sigma}+v_{T}}$ & $M_{\mathrm{IND}}$ & $M_{\mathrm{IND}}$ & $M_{\mathrm{IND}}$ & $M_{\mathrm{IND}}$ \\
\hline
\end{tabular}

Table 6. Relation between the vevs and the mass scales for the different classes. In class A there is no hierarchies in the vevs and thus all scales are equal to $M_{\mathrm{PS}}$. In class $\mathrm{D}$ there are only the scales $M_{\mathrm{PS}}$ and $M_{\mathrm{LR}}$.

\begin{tabular}{|ll|cccccc|}
\hline & Name & $\# h$ & $\# F$ & $\# \Phi$ & $\# \Sigma$ & $\# E$ & $\# T_{L / R}$ \\
\hline SUSY models: & $\mathrm{Em}$ & 0 & 0 & 1 & 1 & 0 & 0 \\
& $\mathrm{Fm}$ & 0 & 0 & 1 & 0 & 0 & 1 \\
& $\mathrm{Es} / \mathrm{Fs}$ & 1 & 1 & 1 & 1 & 1 & 1 \\
& $\mathrm{Ee} / \mathrm{Fe}$ & 3 & 3 & 3 & 3 & 1 & 1 \\
& $\mathrm{Ff}$ & 1 & 3 & 1 & 1 & 1 & 1 \\
& $\mathrm{Bm} / \mathrm{Cm}$ & 0 & 0 & 1 & 1 & 0 & 1 \\
& $\mathrm{Bs} / \mathrm{Cs}$ & 1 & 1 & 1 & 1 & 1 & 1 \\
& $\mathrm{Be} / \mathrm{Ce}$ & 3 & 3 & 1 & 1 & 1 & 1 \\
& $\mathrm{~B} 199$ & 3 & 3 & 1 & 3 & 0 & 1 \\
& $\mathrm{C} 211$ & 3 & 3 & 3 & 3 & 0 & 1 \\
\hline Non-SUSY models: & $\mathrm{E} 289$ & 3 & 3 & 1 & 1 & 0 & 0 \\
& $\mathrm{~F} 213$ & 1 & 3 & 3 & 3 & 1 & 1 \\
& $\mathrm{~B} 53$ & 1 & 3 & 1 & 1 & 3 & 1 \\
& $\mathrm{C} 45$ & 1 & 1 & 3 & 3 & 1 & 1 \\
\hline
\end{tabular}

Table 7. Field content and multiplicities for the discussed models in this paper.

by a subscript that indicates the symmetry which is broken at this stage. Nevertheless, these are of course related to the vevs discussed before, but this relation depends on the class one is discussing. We show this relation explicitly in table 6 .

\section{Model naming scheme}

The global naming convention is laid out at the end of section 3. For all configurations of type $\mathrm{g}$ we use a numerical naming scheme. The numbers follow an internal numbering given by the structure of our Mathematica file. This file is available by the authors upon request.

Table 7 displays the connection between the multiplicities of the different fields present below the Planck scale and the model names used in this paper. 


\section{Specific models}

Among the models that are consistent with unification, there is a subset where further interesting conditions are met simultaneously. In this appendix, we list all models within our framework that (i) show complete GCU at the Planck scale, taken as $M_{\text {Planck }}=10^{18.2} \mathrm{GeV}$; (ii) predict new "exotic" particles at accessible energies $M_{\text {low }} \sim 10 \mathrm{TeV}$; and (iii) exhibit a right-handed neutrino scale in the range $10^{12} \mathrm{GeV}<M_{N_{R}}<10^{14} \mathrm{GeV}$.

Class: F (supersymmetric)

\begin{tabular}{|c|cccccc|cc|}
\hline Model & $\# \mathrm{~h}$ & $\# \mathrm{~F}$ & $\# \Phi$ & $\# \Sigma$ & $\# \mathrm{E}$ & $\# \mathrm{~T}$ & $M_{\mathrm{LR}}[\mathrm{GeV}]$ & $M_{\mathrm{PS}}[\mathrm{GeV}]$ \\
F41 & 0 & 1 & 1 & 1 & 1 & 1 & $\sim 9 \times 10^{11}$ & $\sim 6 \times 10^{14}$ \\
F186 & 1 & 3 & 1 & 1 & 1 & 3 & $\sim 7 \times 10^{12}$ & $\sim 8 \times 10^{14}$ \\
F262 & 3 & 1 & 1 & 3 & 0 & 0 & $\sim 1 \times 10^{11}$ & $\sim 3 \times 10^{14}$ \\
\hline
\end{tabular}

\section{Class: B (supersymmetric)}

\begin{tabular}{|c|cccccc|ccc|}
\hline Model & $\# \mathrm{~h}$ & $\# \mathrm{~F}$ & $\# \Phi$ & $\# \Sigma$ & $\# \mathrm{E}$ & $\# \mathrm{~T}$ & $M_{\mathrm{MSSM}}[\mathrm{GeV}]$ & $M_{\mathrm{LR}}[\mathrm{GeV}]$ & $M_{\mathrm{PS}}[\mathrm{GeV}]$ \\
B19 & 0 & 0 & 3 & 3 & 0 & 1 & $3 \times 10^{10}-3 \times 10^{13}$ & $31 \times 10^{13}-3 \times 10^{15}$ & $2 \times 10^{15}-8 \times 10^{15}$ \\
B115 & 1 & 1 & 3 & 3 & 0 & 1 & $1 \times 10^{11}-3 \times 10^{13}$ & $3 \times 10^{13}-3 \times 10^{15}$ & $2 \times 10^{15}-6 \times 10^{15}$ \\
B199 & 3 & 3 & 1 & 3 & 0 & 1 & $7 \times 10^{11}-1 \times 10^{12}$ & $2 \times 10^{13}-2 \times 10^{14}$ & $3 \times 10^{15}-4 \times 10^{15}$ \\
\hline
\end{tabular}

Class: C (supersymmetric)

\begin{tabular}{|c|cccccc|ccc|}
\hline Model & $\# \mathrm{~h}$ & $\# \mathrm{~F}$ & $\# \Phi$ & $\# \Sigma$ & $\# \mathrm{E}$ & $\# \mathrm{~T}$ & $M_{\mathrm{MSSM}}[\mathrm{GeV}]$ & $M_{\mathrm{PS}}[\mathrm{GeV}]$ & $M_{\mathrm{QL}}[\mathrm{GeV}]$ \\
C43 & 1 & 1 & 3 & 3 & 0 & 1 & $4 \times 10^{10}-3 \times 10^{12}$ & $7 \times 10^{15}-3 \times 10^{16}$ & $7 \times 10^{15}-7 \times 10^{17}$ \\
C53 & 1 & 3 & 1 & 1 & 3 & 1 & $2 \times 10^{12}-1 \times 10^{14}$ & $3 \times 10^{16}-6 \times 10^{16}$ & $1 \times 10^{17}-2 \times 10^{18}$ \\
C127 & 3 & 3 & 1 & 3 & 0 & 1 & $4 \times 10^{11}-4 \times 10^{12}$ & $5 \times 10^{15}-8 \times 10^{15}$ & $5 \times 10^{15}-5 \times 10^{17}$ \\
\hline
\end{tabular}

Non-supersymmetric

\begin{tabular}{|c|cccccc|ccc|}
\hline Model & $\# \mathrm{~h}$ & $\# \mathrm{~F}$ & $\# \Phi$ & $\# \Sigma$ & $\# \mathrm{E}$ & $\# \mathrm{~T}$ & $M_{\mathrm{SM}}[\mathrm{GeV}]$ & $M_{\mathrm{PS}}[\mathrm{GeV}]$ & $M_{\mathrm{QL}}[\mathrm{GeV}]$ \\
E46 & 0 & 1 & 1 & 3 & 0 & 0 & $4 \times 10^{12}$ & $3 \times 10^{14}$ & - \\
E73 & 0 & 3 & 1 & 1 & 0 & 0 & $9 \times 10^{10}$ & $6 \times 10^{14}$ & - \\
E87 & 0 & 3 & 1 & 3 & 1 & 3 & $9 \times 10^{10}$ & $6 \times 10^{14}$ & - \\
E89 & 0 & 3 & 1 & 3 & 3 & 1 & $9 \times 10^{10}$ & $6 \times 10^{14}$ & - \\
C193 & 1 & 3 & 1 & 3 & 0 & 1 & $6 \times 10^{10}-3 \times 10^{13}$ & $2 \times 10^{13}-1 \times 10^{14}$ & $3 \times 10^{16}-4 \times 10^{17}$ \\
\hline
\end{tabular}

\section{Acknowledgments}

This work has been supported by a Coordinated Project Grant of the Faculty of Science and Technology, University of Siegen. We thank T. Feldmann, C. Luhn and J. Reuter for discussions and valuable comments on the manuscript. 
Open Access. This article is distributed under the terms of the Creative Commons Attribution License (CC-BY 4.0), which permits any use, distribution and reproduction in any medium, provided the original author(s) and source are credited.

\section{References}

[1] R.N. Mohapatra and J.C. Pati, A natural left-right symmetry, Phys. Rev. D 11 (1975) 2558 [INSPIRE].

[2] G. Senjanović and R.N. Mohapatra, Exact left-right symmetry and spontaneous violation of parity, Phys. Rev. D 12 (1975) 1502 [INSPIRE].

[3] J.C. Pati and A. Salam, Lepton number as the fourth color, Phys. Rev. D 10 (1974) 275 [Erratum ibid. D 11 (1975) 703] [INSPIRE].

[4] J.C. Pati and A. Salam, Unified lepton-hadron symmetry and a gauge theory of the basic interactions, Phys. Rev. D 8 (1973) 1240 [INSPIRE].

[5] H. Georgi and S.L. Glashow, Unity of all elementary particle forces, Phys. Rev. Lett. 32 (1974) 438 [inSPIRE].

[6] H. Fritzsch and P. Minkowski, Unified interactions of leptons and hadrons, Annals Phys. 93 (1975) 193 [INSPIRE].

[7] F. Gürsey, P. Ramond and P. Sikivie, A universal gauge theory model based on $E_{6}$, Phys. Lett. B 60 (1976) 177 [InSPIRE].

[8] M. Malinský, J.C. Romão and J.W.F. Valle, Supersymmetric $\mathrm{SO}(10)$ seesaw mechanism with low B - L scale, Phys. Rev. Lett. 95 (2005) 161801 [hep-ph/0506296] [INSPIRE].

[9] C. Arbeláez, R.M. Fonseca, M. Hirsch and J.C. Romão, Supersymmetric SO(10)-inspired GUTs with sliding scales, Phys. Rev. D 87 (2013) 075010 [arXiv:1301.6085] [INSPIRE].

[10] V. De Romeri, M. Hirsch and M. Malinský, Soft masses in SUSY SO(10) GUTs with low intermediate scales, Phys. Rev. D 84 (2011) 053012 [arXiv:1107.3412] [INSPIRE].

[11] L. Calibbi, M. Frigerio, S. Lavignac and A. Romanino, Flavour violation in supersymmetric $\mathrm{SO}(10)$ unification with a type-II seesaw mechanism, JHEP 12 (2009) 057 [arXiv: 0910.0377] [INSPIRE].

[12] L. Calibbi, L. Ferretti, A. Romanino and R. Ziegler, Gauge coupling unification, the GUT scale and magic fields, Phys. Lett. B 672 (2009) 152 [arXiv:0812.0342] [InSPIRE].

[13] C. Biggio and L. Calibbi, Phenomenology of SUSY SU(5) with type-I+III seesaw, JHEP 10 (2010) 037 [arXiv: 1007.3750] [INSPIRE].

[14] C. Biggio, L. Calibbi, A. Masiero and S.K. Vempati, Postcards from oases in the desert: phenomenology of SUSY with intermediate scales, JHEP 08 (2012) 150 [arXiv:1205.6817] [INSPIRE].

[15] M.R. Buckley and H. Murayama, How can we test seesaw experimentally?, Phys. Rev. Lett. 97 (2006) 231801 [hep-ph/0606088] [INSPIRE].

[16] J.N. Esteves, S. Kaneko, J.C. Romão, M. Hirsch and W. Porod, Dark matter in minimal supergravity with type-II seesaw, Phys. Rev. D 80 (2009) 095003 [arXiv:0907.5090] [INSPIRE]. 
[17] J.N. Esteves, J.C. Romão, M. Hirsch, F. Staub and W. Porod, Supersymmetric type-III seesaw: lepton flavour violating decays and dark matter, Phys. Rev. D 83 (2011) 013003 [arXiv: 1010.6000] [INSPIRE].

[18] J.N. Esteves et al., Dark matter and LHC phenomenology in a left-right supersymmetric model, JHEP 01 (2012) 095 [arXiv:1109.6478] [inSPIRE].

[19] S.K. Majee, M.K. Parida, A. Raychaudhuri and U. Sarkar, Low intermediate scales for leptogenesis in SUSY SO(10) GUTs, Phys. Rev. D 75 (2007) 075003 [hep-ph/0701109] [INSPIRE].

[20] F. Braam, J. Reuter and D. Wiesler, The Pati-Salam supersymmetric SM (PSSSM), AIP Conf. Proc. 1200 (2010) 458 [arXiv: 0909. 3081] [InSPIRE].

[21] W. Kilian and J. Reuter, Unification without doublet-triplet splitting, Phys. Lett. B 642 (2006) 81 [hep-ph/0606277] [INSPIRE].

[22] R. Howl and S.F. King, Planck scale unification in a supersymmetric standard model, Phys. Lett. B 652 (2007) 331 [arXiv:0705.0301] [InSPIRE].

[23] P.S. Bhupal Dev and R.N. Mohapatra, TeV scale inverse seesaw in $\mathrm{SO}(10)$ and leptonic non-unitarity effects, Phys. Rev. D 81 (2010) 013001 [arXiv:0910.3924] [INSPIRE].

[24] M. Lindner and M. Weiser, Gauge coupling unification in left-right symmetric models, Phys. Lett. B 383 (1996) 405 [hep-ph/9605353] [INSPIRE].

[25] C. Arbeláez, M. Hirsch, M. Malinský and J.C. Romão, LHC-scale left-right symmetry and unification, Phys. Rev. D 89 (2014) 035002 [arXiv:1311.3228] [INSPIRE].

[26] F. Siringo, Grand unification in the minimal left-right symmetric extension of the standard model, Phys. Part. Nucl. Lett. 10 (2013) 94 [arXiv:1208.3599] [InSPIRE].

[27] C.S. Aulakh, B. Bajc, A. Melfo, A. Rasin and G. Senjanović, Intermediate scales in supersymmetric GUTs: the survival of the fittest, Phys. Lett. B 460 (1999) 325 [hep-ph/9904352] [INSPIRE].

[28] C.S. Aulakh, B. Bajc, A. Melfo, A. Rasin and G. Senjanović, SO(10) theory of R-parity and neutrino mass, Nucl. Phys. B 597 (2001) 89 [hep-ph/0004031] [InSPIRE].

[29] Particle Data Group collaboration, J. Beringer et al., Review of particle physics, Phys. Rev. D 86 (2012) 010001 [INSPIRE].

[30] S. Weinberg, Baryon and lepton nonconserving processes, Phys. Rev. Lett. 43 (1979) 1566 [INSPIRE].

[31] P. Minkowski, $\mu \rightarrow e \gamma$ at a rate of one out of 1-billion muon decays?, Phys. Lett. B 67 (1977) 421 [INSPIRE].

[32] H. Georgi, Towards a grand unified theory of flavor, Nucl. Phys. B 156 (1979) 126 [INSPIRE].

[33] F. del Aguila and L.E. Ibáñez, Higgs bosons in $\mathrm{SO}(10)$ and partial unification, Nucl. Phys. B 177 (1981) 60 [InSPIRE].

[34] R.N. Mohapatra and G. Senjanović, Higgs boson effects in grand unified theories, Phys. Rev. D 27 (1983) 1601 [inSPIRE].

[35] S. Dimopoulos and H.M. Georgi, Extended survival hypothesis and fermion masses, Phys. Lett. B 140 (1984) 67 [InSPIRE]. 
[36] C.S. Aulakh and R.N. Mohapatra, Implications of supersymmetric $\mathrm{SO}(10)$ grand unification, Phys. Rev. D 28 (1983) 217 [InSPIRE].

[37] R. Slansky, Group theory for unified model building, Phys. Rept. 79 (1981) 1 [InSPIRE].

[38] H.P. Nilles, Supersymmetry, supergravity and particle physics, Phys. Rept. 110 (1984) 1 [INSPIRE].

[39] H.E. Haber and G.L. Kane, The search for supersymmetry: probing physics beyond the standard model, Phys. Rept. 117 (1985) 75 [INSPIRE].

[40] J. Wess and B. Zumino, A Lagrangian model invariant under supergauge transformations, Phys. Lett. B 49 (1974) 52 [InSPIRE].

[41] M.T. Grisaru, W. Siegel and M. Roček, Improved methods for supergraphs, Nucl. Phys. B 159 (1979) 429 [inSPIRE].

[42] C.S. Aulakh, K. Benakli and G. Senjanović, Reconciling supersymmetry and left-right symmetry, Phys. Rev. Lett. 79 (1997) 2188 [hep-ph/9703434] [INSPIRE].

[43] C.S. Aulakh, A. Melfo and G. Senjanović, Minimal supersymmetric left-right model, Phys. Rev. D 57 (1998) 4174 [hep-ph/9707256] [INSPIRE].

[44] J.R. Ellis, J.F. Gunion, H.E. Haber, L. Roszkowski and F. Zwirner, Higgs bosons in a nonminimal supersymmetric model, Phys. Rev. D 39 (1989) 844 [INSPIRE].

[45] H.P. Nilles, M. Srednicki and D. Wyler, Weak interaction breakdown induced by supergravity, Phys. Lett. B 120 (1983) 346 [inSPIRE].

[46] R.N. Mohapatra and G. Senjanović, Neutrino mass and spontaneous parity violation, Phys. Rev. Lett. 44 (1980) 912 [InSPIRE].

[47] M. Gell-Mann, P. Ramond and R. Slansky, Complex spinors and unified theories, Conf. Proc. C $\mathbf{7 9 0 9 2 7}$ (1979) 315 [arXiv:1306.4669] [INSPIRE].

[48] D.R.T. Jones, Two-loop $\beta$ function for a $G_{1} \times G_{2}$ gauge theory, Phys. Rev. D 25 (1982) 581 [INSPIRE]. 